\title{
Complex projective towers and their cohomological rigidity up to dimension six
}

\section{Shintaro KUROKI and DongYoup SUH}

\begin{tabular}{|c|c|}
\hline Citation & OCAMI Preprint Series \\
\hline Issue Date & 2012 \\
\hline Type & Preprint \\
\hline Textversion & Author \\
\hline Rights & For personal use only. No other uses without permission. \\
\hline Relation & $\begin{array}{l}\text { This is a pre-print of an article published in Proceedings of the Steklov } \\
\text { Institute of Mathematics. The final authenticated version is available } \\
\text { online at: } \underline{\text { https://doi.org/10.1134/S0081543814060170 }}\end{array}$ \\
\hline
\end{tabular}

From: Osaka City University Advanced Mathematical Institute http://www.sci.osaka-cu.ac.jp/OCAMI/publication/preprint/preprint.html 


\title{
Complex projective towers and their cohomological rigidity up to dimension six
}

\author{
Shintarô KUROKI and DongYoup SUH
}

Dedicated to Professor Victor Buchstaber on his 70th birthday.

\begin{abstract}
A complex projective tower or simply a $\mathbb{C} P$-tower is an iterated complex projective fibrations starting from a point. In this paper we classify all 6-dimensional $\mathbb{C} P$-towers up to diffeomorphism, and as a consequence, we show that all such manifolds are cohomologically rigid, i.e., they are completely determined up to diffeomorphism by their cohomology rings.
\end{abstract}

\section{Contents}

1. Introduction

2. Some preliminaries 3

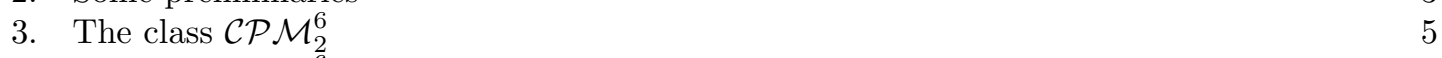

4. The class $\mathcal{C P M}_{3}^{6} \quad 8$

$\begin{array}{ll}\text { Acknowledgments } & 20\end{array}$

$\begin{array}{ll}\text { References } & 20\end{array}$

\section{Introduction}

An iterated complex projective fibration is a sequence of fibrations

$$
C_{m} \stackrel{\pi_{m}}{\longrightarrow} C_{m-1} \stackrel{\pi_{m-1}}{\longrightarrow} \cdots \stackrel{\pi_{2}}{\longrightarrow} C_{1} \stackrel{\pi_{1}}{\longrightarrow} C_{0}=\{\text { a point }\}
$$

where $\pi_{i+1}: C_{i+1} \rightarrow C_{i}$ is a fibration with $\mathbb{C} P^{n_{i}}$ for some $n_{i} \in \mathbb{N}$ as its fiber for $i=0, \ldots, m-1$. In this paper, we study the topology of the following special type of iterated complex projective fibrations: a complex projective tower (or simply a $\mathbb{C} P$-tower) of height $m$ is an iterated complex projective fibration in (1.1) where $C_{i+1}=P\left(\xi_{i}\right)$ is the projectivization of a complex vector bundle $\xi_{i}$ over $C_{i}$. It is also called an $m$-stage $\mathbb{C} P$-tower. We call each $C_{i}$ the $i$ th stage of the tower, and the top stage manifold $C_{m}$ is simply called a $\mathbb{C} P$-manifold.

If each complex vector bundle $\xi_{i}$ in a $\mathbb{C} P$-tower is a Whitney sum of complex line bundles, such $\mathbb{C} P$-tower (resp. $\mathbb{C} P$-manifold) is known as a generalized Bott tower (resp. generalized Bott manifold) (see $[\mathbf{C M S 1 0}]$ ). If each $\xi_{i}$ is a sum of just two complex line bundles, then it is a Bott tower (resp. Bott manifold), introduced in [BoSa] (also see [GrKa]). In particular, every Hirzebruch surfaces is a 2-stage Bott manifold.

The first author was partially supported by the JSPS Institutional Program for Young Researcher Overseas Visits " Promoting international young researchers in mathematics and mathematical sciences led by OCAMI" and the JSPS Strategic Young Researcher Overseas Visits Program for Accelerating Brain Circulation "Deepening and Evolution of Mathematics and Physics, Building of International Network Hub based on OCAMI". The second author was supported in part by Basic Science Research Program through the National Research Foundation of Korea(NRF) funded by the Ministry of Education(2013R1A1A2007780). 
Any $n$-dimensional generalized Bott manifolds have effective complex $n$-torus actions so that they have structures of toric manifolds. On the other hand, even though the Milnor surface $H_{i j} \subset \mathbb{C} P^{i} \times \mathbb{C} P^{j}$ has the structure of a 2-stage $\mathbb{C} P$-tower as is explained in Example 2.3, it does not admit any toric manifold structure when $i$ and $j$ are sufficiently large, see Remark 2.4. Therefore $\mathbb{C} P$-manifolds do not have toric manifold structures, in general.

Let $\mathcal{M}$ be a class of diffeomorphism classes of manifolds, and let $\mathbf{H}^{*} \mathcal{M}$ be the isomorphism classes of cohomology rings of manifolds in $\mathcal{M}$. Let $H^{*}: \mathcal{M} \rightarrow \mathbf{H}^{*} \mathcal{M}$ be the map defined by $M \in \mathcal{M} \mapsto H^{*}(M ; \mathbb{Z})$. The class $\mathcal{M}$ is said to be cohomologically rigid if the map $H^{*}$ is bijective. One of the open questions on cohomological rigidity is whether the class of toric manifolds are cohomologically rigid. Even though there is no negative answer to the question so far, the class of toric manifolds is too broad to handle in order to get the positive answer to the question. So we need to restrict our attention to a smaller class of manifolds. Accordingly, one might ask whether the class of diffeomorphism classes of (generalized) Bott manifolds are cohomologically rigid. There are some partial answers to the question in $[$ CMS10, CPS, MaPa], and we refer the reader to [CMS11] for the summary of the most recent developments about the question. In particular, the class of $m$-stage Bott manifolds for $n \leq 4$ (see [Ch] and [CMS10]) and the class of 2-stage generalized Bott manifolds (see [CMS10]) are cohomologically rigid.

Since the class of $\mathbb{C} P$-manifolds contains the class of generalized Bott manifolds, one might ask the cohomological rigidity question to the class of $\mathbb{C} P$-manifolds. Let $\mathcal{C P} \mathcal{M}^{2 n}$ (resp. $\mathcal{C P} \mathcal{M}_{m}^{2 n}$ ) be the class of diffeomorphism classes of (resp. $m$-stage) $2 n$-dimensional $\mathbb{C} P$-manifolds. The goal of this paper is to show that the class $\mathcal{C P} \mathcal{M}^{2 n}$ for $n \leq 3$ is cohomologically rigid. This is done by classifying all the members of $\mathcal{C P} \mathcal{M}_{m}^{2 n}$ for $1 \leq m \leq n \leq 3$ and showing that their cohomology rings are all distinct. However the class of $\mathbb{C} P$-manifolds is not cohomologically rigid, in general. In fact, in $[\mathbf{K u S u}]$ we will show that that $\mathcal{C P} \mathcal{M}^{8}$ is not cohomologically rigid.

We now describe our classification results. Note that the only 2-dimensional $\mathbb{C} P$-manifold is $\mathbb{C} P^{1}$, i.e., $\mathcal{C P} \mathcal{M}^{2}$ is cohomologically rigid and

$$
\mathcal{C P} \mathcal{M}^{2}=\left\{\mathbb{C} P^{1}\right\}
$$

Any 4-dimensional $\mathbb{C} P$-manifold is either $\mathbb{C} P^{2}$ or a 2-stage $\mathbb{C} P$-manifold which is in fact nothing but a Hirzebruch surface as we have stated above. So they are either $H_{0}:=\mathbb{C} P^{1} \times \mathbb{C} P^{1}$ or $H_{1}:=\mathbb{C} P^{2} \# \overline{\mathbb{C} P^{2}}$. Since their cohomology rings are not isomorphic, $\mathcal{C P} \mathcal{M}^{4}$ is cohomologically rigid and

$$
\mathcal{C P} \mathcal{M}^{4}=\left\{\mathbb{C} P^{2}, H_{0}, H_{1}\right\}
$$

For 6-dimensional $\mathbb{C} P$-manifolds, we have to consider one-stage $\mathbb{C} P$-manifold which is $\mathbb{C} P^{3}$, twostage $\mathbb{C} P$-manifolds, and three-stage $\mathbb{C} P$-manifolds separately. For two-stage 6-dimensional $\mathbb{C} P$ manifolds, there are two cases; the cases when the first stages are $C_{1}=\mathbb{C} P^{1}$ or $C_{1}=\mathbb{C} P^{2}$. When $C_{1}=\mathbb{C} P^{1}$, then $C_{2}=P(\xi)$ where $\xi$ is a sum of three complex line bundles because of the dimensional reason. Therefore, $C_{2}$ must be a two-stage generalized Bott manifold, which is completely determined up to diffeomorphism in [CMS10]. In fact, there are only three diffeomorphism types $P\left(\gamma_{1}^{k} \oplus \epsilon \oplus \epsilon\right) \rightarrow \mathbb{C} P^{1}$ for $k=0,1,2$, where $\gamma_{1}$ is the tautological line bundle over $\mathbb{C} P^{1}$.

For two-stage 6-dimensional $\mathbb{C} P$-manifolds with $C_{1}=\mathbb{C} P^{2}$, the second stage is $C_{2}=P(\xi)$, where $\xi$ is a rank 2-complex vector bundle over $\mathbb{C} P^{2}$, which is determined by its Chern classes $c_{1} \in H^{2}\left(\mathbb{C} P^{2}\right) \simeq \mathbb{Z}$ and $c_{2} \in H^{4}\left(\mathbb{C} P^{2}\right) \simeq \mathbb{Z}$. It is proved that the diffeomorphism types of such $\mathbb{C} P$-manifolds are $P\left(\eta_{(0, \alpha)}\right) \rightarrow \mathbb{C} P^{2}$ and $P\left(\eta_{(1, \alpha)}\right) \rightarrow \mathbb{C} P^{2}$ for $\alpha \in H^{4}\left(\mathbb{C} P^{2}\right) \simeq \mathbb{Z}$, where $\eta_{(s, \alpha)}$ is a $\mathbb{C}$-vector bundle over $\mathbb{C} P^{2}$ whose Chern classes are $\left(c_{1}, c_{2}\right)=(s, \alpha)$.

For three-stage $\mathbb{C} P$-manifolds $C_{3} \rightarrow C_{2} \rightarrow C_{1}$, there are two cases, i.e., when $C_{2}=H_{0}=$ $\mathbb{C} P^{1} \times \mathbb{C} P^{1}$ and $C_{2}=H_{1}=\mathbb{C} P^{2} \# \overline{\mathbb{C} P^{2}}$. Then $C_{3}=P(\xi)$ where $\xi$ is a complex 2-dimensional vector bundle over $C_{2}$. Again, it is proved in Lemma 4.1 that $\xi$ is classified by its Chern classes $c_{1}$ and $c_{2}$. Let $\eta_{(s, r, \alpha)}$ (resp. $\left.\xi_{(s, r, \alpha)}\right)$ be the complex 2-dimensional bundle over $\mathbb{C} P^{1} \times \mathbb{C} P^{1}\left(\right.$ resp. $\left.\mathbb{C} P^{2} \# \overline{\mathbb{C} P^{2}}\right)$ whose first Chern class $c_{1}\left(\eta_{(s, r, \alpha)}\right)=(s, r) \in H^{2}\left(\mathbb{C} P^{1} \times \mathbb{C} P^{1}\right) \simeq \mathbb{Z} \oplus \mathbb{Z}\left(\operatorname{resp} . c_{1}\left(\xi_{(s, r, \alpha)}\right)=(s, r) \in\right.$ $\left.H^{2}\left(\mathbb{C} P^{2} \# \overline{\mathbb{C} P^{2}}\right)\right)$ and the second Chern class $c_{2}\left(\eta_{(s, r, \alpha)}\right)=\alpha \in H^{4}\left(\mathbb{C} P^{1} \times \mathbb{C} P^{1}\right) \simeq \mathbb{Z}$ (resp. 
$\left.c_{2}\left(\xi_{(s, r, \alpha)}\right)=\alpha \in H^{4}\left(\mathbb{C} P^{2} \# \overline{\mathbb{C} P^{2}}\right)\right)$. Then, it is proved that all diffeomorphism types of threestage $\mathbb{C} P$-manifolds are $P\left(\zeta_{(s, r, \alpha)}\right) \rightarrow H_{0}$ and $P\left(\xi_{(s, r, \alpha)}\right) \rightarrow H_{1}$ for $\alpha \in \mathbb{Z}$ and $(s, r)=(0,0),(1,0)$ or $(1,1)$.

We thus have the following classification result of 6 -dimensional $\mathbb{C} P$-manifolds.

THEOREM 1.1. The class $\mathcal{C} \mathcal{P} \mathcal{M}^{6}$ consists of diffeomorphism classes of the following distinct manifolds:

- $\mathbb{C} P^{3}$;

- $P\left(\gamma_{1}^{k} \oplus \epsilon \oplus \epsilon\right) \rightarrow \mathbb{C} P^{1}$ for $k=0,1,2$;

- $P\left(\eta_{(0, \alpha)}\right) \rightarrow \mathbb{C} P^{2}$ for $\alpha \in \mathbb{Z} \backslash\{0\}$;

- $P\left(\eta_{(1, \alpha)}\right) \rightarrow \mathbb{C} P^{2}$ for $\alpha \in \mathbb{Z}$;

- $P\left(\zeta_{(0,0, \alpha)}\right) \rightarrow H_{0}$ for $\alpha \in \mathbb{Z}_{\geq 0}$;

- $P\left(\zeta_{(1,0, \alpha)}\right) \rightarrow H_{0}$ for $\alpha \in \mathbb{Z}_{\geq 0}$;

- $P\left(\zeta_{(1,1, \alpha)}\right) \rightarrow H_{0}$ for $\alpha \in \mathbb{N}$;

- $P\left(\xi_{(0,0, \alpha)}\right) \rightarrow H_{1}$ for $\alpha \in \mathbb{N}$;

- $P\left(\xi_{(1,0, \alpha)}\right) \rightarrow H_{1}$ for $\alpha \in \mathbb{Z}_{\geq 0}$;

- $P\left(\xi_{(1,1, \alpha)}\right) \rightarrow H_{1}$ for $\alpha \in \mathbb{Z}$,

where $H_{0}:=\mathbb{C} P^{1} \times \mathbb{C} P^{1}, H_{1}:=\mathbb{C} P^{2} \# \overline{\mathbb{C} P^{2}}$, and the symbols $\mathbb{N}, \mathbb{Z}_{\geq 0}$ and $\mathbb{Z}$ represent natural numbers, non-negative integers and integers, respectively. In other wards, any 6 -dimensional $\mathbb{C} P$ manifold is diffeomorphic to one of the manifolds as above.

Since the cohomology rings of the manifolds in Theorem 1.1 are mutually non-isomorphic, we have the following corollary on cohomological rigidity of $\mathbb{C} P$-manifolds.

Corollary 1.2. Let $M_{1}$ and $M_{2}$ be two $\mathbb{C} P$-manifolds of dimension less than or equal to 6 . Then, $M_{1}$ and $M_{2}$ are diffeomorphic if and only if their cohomology rings $H^{*}\left(M_{1}\right)$ and $H^{*}\left(M_{2}\right)$ are isomorphic. In other wards, $\mathcal{C P} \mathcal{M}^{2 n}$ for $n \leq 3$ is cohomologically rigid.

This corollary is a generalization of the cohomological rigidity theorem for Bott manifolds up to dimension less than or equal to 6 proved in [CMS10].

The organization of this paper is as follows. In Section 2, we prepare some basics and some examples. In Section 3, we classify 6-dimensional $\mathbb{C} P$-manifolds with height 2 up to diffeomorphism. In Section 4, we classify 6-dimensional $\mathbb{C} P$-manifolds with height 3 . Theorem 1.1 is proved as a consequence of the classification.

\section{Some preliminaries}

In this section, we prepare some basic facts which will be used in later sections. Let $\xi$ be an $n$-dimensional complex vector bundle over a topological space $X$, and let $P(\xi)$ denote its projectivization. Then the Borel-Hirzebruch formula in [BoHi] says

$$
H^{*}(P(\xi) ; \mathbb{Z}) \simeq H^{*}(X ; \mathbb{Z})[x] /\left\langle x^{n+1}+\sum_{i=1}^{n}(-1)^{i} c_{i}\left(\pi^{*} \xi\right) x^{n+1-i}\right\rangle
$$

where $\pi^{*} \xi$ is the pull-back of $\xi$ along $\pi: P(\xi) \rightarrow X$ and $c_{i}\left(\pi^{*} \xi\right)$ is the $i$ th Chern class of $\pi^{*} \xi$. Here $x$ can be viewed as the first Chern class of the canonical line bundle over $P(\xi)$, i.e., the complex 1-dimensional sub-bundle $\gamma_{\xi}$ in $\pi^{*} \xi \rightarrow P(\xi)$ such that the restriction $\left.\gamma_{\xi}\right|_{\pi^{-1}(a)}$ is the canonical line bundle over $\pi^{-1}(a) \cong \mathbb{C} P^{n-1}$ for all $a \in X$. Therefore $\operatorname{deg} x=2$. Since it is well-known that the induced homomorphism $\pi^{*}: H^{*}(X ; \mathbb{Z}) \rightarrow H^{*}(P(\xi) ; \mathbb{Z})$ is injective, we often abuse the notation $c_{i}\left(\pi^{*} \xi\right)$ by $c_{i}(\xi)$.

We apply the formula $(2.1)$ to an $m$-stage $\mathbb{C} P$-manifold

$$
C_{m} \stackrel{\pi_{m}}{\longrightarrow} C_{m-1} \stackrel{\pi_{m-1}}{\longrightarrow} \cdots \stackrel{\pi_{2}}{\longrightarrow} C_{1} \stackrel{\pi_{1}}{\longrightarrow} C_{0}=\{\text { a point }\}
$$


with $C_{i}=P\left(\xi_{i-1}\right)$, to get the following isomorphisms.

$$
\begin{aligned}
H^{*}\left(C_{m} ; \mathbb{Z}\right) & \simeq H^{*}\left(C_{m-1} ; \mathbb{Z}\right)\left[x_{m}\right] /\left\langle x_{m}^{n_{m}+1}+\sum_{i=1}^{n_{m}}(-1)^{i} c_{i}\left(\xi_{m-1}\right) x_{m}^{n_{m}+1-i}\right\rangle \\
& \simeq H^{*}\left(C_{m-2} ; \mathbb{Z}\right)\left[x_{m-1}, x_{m}\right] /\left\langle x_{k}^{n_{k}+1}+\sum_{i=1}^{n_{k}}(-1)^{i} c_{i}\left(\xi_{k}\right) x_{k}^{n_{k}+1-i} \mid k=m-1, m\right\rangle \\
& \vdots \\
& \simeq \mathbb{Z}\left[x_{1}, \ldots, x_{m}\right] /\left\langle x_{k}^{n_{k}+1}+\sum_{i=1}^{n_{k}}(-1)^{i} c_{i}\left(\xi_{k}\right) x_{k}^{n_{k}+1-i} \mid k=1, \cdots, m\right\rangle .
\end{aligned}
$$

This formula gives all elements in the class $\mathbf{H}^{*} \mathcal{C} \mathcal{P} \mathcal{M}^{2 n}$.

In order to prove the main theorem, we often use the following lemmas.

LEMma 2.1. Let $\gamma$ be any line bundle over $M$, and let $P(\xi)$ be the projectivization of a complex vector bundle $\xi$ over $M$. Then, $P(\xi)$ is diffeomorphic to $P(\xi \otimes \gamma)$.

Proof. By the definition of the projectivization of a complex vector bundle, the statement follows immediately.

Lemma 2.2. Let $\gamma$ be a complex line bundle, and let $\xi$ be a 2-dimensional complex vector bundle over a manifold $M$. Then the Chern classes of the tensor product $\xi \otimes \gamma$ are as follows.

$$
\begin{aligned}
& c_{1}(\xi \otimes \gamma)=c_{1}(\xi)+2 c_{1}(\gamma) ; \\
& c_{2}(\xi \otimes \gamma)=c_{1}(\gamma)^{2}+c_{1}(\gamma) c_{1}(\xi)+c_{2}(\xi) .
\end{aligned}
$$

Proof. Let us consider the following pull-back diagram:

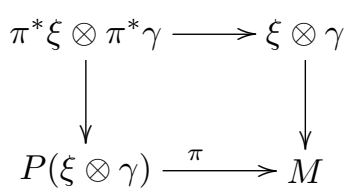

Let $\varphi: P(\xi \otimes \gamma) \rightarrow P(\xi)$ be the diffeomorphism from Lemma 2.1, and let $\pi_{\xi}: P(\xi) \rightarrow M$ be the projection of the fibration. Then we can see easily that $\pi=\pi_{\xi} \circ \varphi$. Taking the canonical line bundle $\gamma_{\xi}$ in $\pi_{\xi}^{*} \xi$, we may regard $\pi_{\xi}^{*} \xi \equiv \gamma_{\xi} \oplus \gamma_{\xi}^{\perp}$, where $\gamma_{\xi}^{\perp}$ is the normal (line) bundle of $\gamma_{\xi}$ in $\pi_{\xi}^{*} \xi$. By using the decomposition $\pi=\pi_{\xi} \circ \varphi$, we have the following equation:

$$
\begin{aligned}
\pi^{*} c(\xi \otimes \gamma) & =c\left(\varphi^{*} \gamma_{\xi} \otimes \pi^{*}(\gamma)\right) c\left(\varphi^{*} \gamma_{\xi}^{\perp} \otimes \pi^{*}(\gamma)\right) \\
& =\left(1+\varphi^{*} c_{1}\left(\gamma_{\xi}\right)+\pi^{*} c_{1}(\gamma)\right)\left(1+\varphi^{*} c_{1}\left(\gamma_{\xi}^{\perp}\right)+\pi^{*} c_{1}(\gamma)\right) .
\end{aligned}
$$

Because $\pi^{*} c_{1}(\xi)=\varphi^{*} c_{1}\left(\gamma_{\xi}\right)+\varphi^{*} c_{1}\left(\gamma_{\xi}^{\perp}\right)$ and $\pi^{*} c_{2}(\xi)=\varphi^{*} c_{1}\left(\gamma_{\xi}\right) \varphi^{*} c_{1}\left(\gamma_{\xi}^{\perp}\right)$, we have

$$
\begin{aligned}
& \pi^{*} c_{1}(\xi \otimes \gamma)=\pi^{*} c_{1}(\xi)+2 \pi^{*} c_{1}(\gamma) ; \\
& \pi^{*} c_{2}(\xi \otimes \gamma)=\pi^{*} c_{2}(\xi)+\pi^{*} c_{1}(\xi) \pi^{*} c_{1}(\gamma)+\pi^{*} c_{1}(\gamma)^{2} .
\end{aligned}
$$

As is well-known, $\pi^{*}: H^{*}(M) \rightarrow H^{*}(P(\xi \otimes \gamma))$ is injective. Hence we have the formula in the lemma.

We now give an example of $\mathcal{C P} \mathcal{M}_{2}^{2 n}$.

EXAmple 2.3. The Milnor hypersurface $H_{i, j} \subset \mathbb{C} P^{i} \times \mathbb{C} P^{j}, 1 \leq i \leq j$ is defined by the following equation (see [BuPa, Example 5.39]):

$$
H_{i, j}=\left\{\left[z_{0}: \cdots: z_{i}\right] \times\left[w_{0}: \cdots: w_{j}\right] \in \mathbb{C} P^{i} \times \mathbb{C} P^{j} \mid \sum_{q=0}^{i} z_{q} w_{q}=0\right\} .
$$

We can show easily that the natural projection onto the first coordinate of $H_{i, j}$ gives the structure of a $\mathbb{C} P^{j-1}$-bundle over $\mathbb{C} P^{i}$. Moreover, by the proof in [BuPa, Theorem 5.39], this bundle may be regarded as the projectivization of $\gamma^{\perp} \subset \epsilon^{j+1}$, where $\epsilon^{j+1}$ is the trivial $\mathbb{C}^{j+1}$-bundle over $\mathbb{C} P^{i}$ 
and $\gamma^{\perp}$ is the normal bundle of the canonical line bundle $\gamma$ over $\mathbb{C} P^{i}$ in $\epsilon^{j+1}$. Therefore, the Milnor hypersurface admits the structure of a $\mathbb{C} P$-manifold with height 2.

REMARK 2.4. As is well-known, the Milnor hypersurface $H_{i, j}$ with $i \geq 2$ does not admit the structure of a toric manifold (see e.g. [BuPa]). On the other hand, $H_{1, j} \rightarrow \mathbb{C} P^{1}$ is a toric manifold.

REMARK 2.5. The structures of fibre bundles of projectivization of complex $m$-dimensional vector bundles are classified by homotopy classes of maps from the base space of the bundle to the classifying space $B P U(m)$ of the projective unitary group $P U(m)$, i.e., $P U(m)=U(m) / T^{1}$ where $T^{1}$ is the diagonal subgroup of the unitary group $U(m)$. Therefore a $\mathbb{C} P$-tower is a special kind of iterated complex projective fibrations.

\section{The class $\mathcal{C P} \mathcal{M}_{2}^{6}$}

Let $M \in \mathcal{C} \mathcal{P} \mathcal{M}_{m}^{6}$ be an $m$-stage 6-dimensional $\mathbb{C} P$-manifold. Then, the height $m \leq 3$. In particular, if $m=1$, then $M$ is diffeomorphic to $\mathbb{C} P^{3}$, i.e., $\mathcal{C P} \mathcal{M}_{1}^{6}=\left\{\mathbb{C} P^{3}\right\}$. Therefore, it is enough to analyze the case when the height $m$ is 2 and 3 . In this section, we focus on the classification of 6 -dimensional $\mathbb{C} P$-manifolds of height 2 .

To state the main theorem of this section, we first set up some notation. Let $\gamma_{i}$ denote the tautological line bundle over $\mathbb{C} P^{i}$, and let $x$ denote the generator $-c_{1}\left(\gamma_{2}\right) \in H^{2}\left(\mathbb{C} P^{2}\right)$. Let $\eta_{(s, \alpha)}$ as the complex 2-dimensional vector bundle over $\mathbb{C} P^{2}$ whose total Chern class is $1+s x+\alpha x^{2}$ for $s, \alpha \in \mathbb{Z}$, let $P\left(\eta_{(s, \alpha)}\right)$ be its projectivization. We now state the main theorem of this section.

TheOREM 3.1. The class $\mathcal{C} \mathcal{P} \mathcal{M}_{2}^{6}$ consists of the following distinct $\mathbb{C P}$-manifolds.

$$
\begin{aligned}
& P\left(\gamma_{1} \oplus \epsilon \oplus \epsilon\right) \longrightarrow \mathbb{C} P^{1} ; \\
& P\left(\gamma_{1}^{2} \oplus \epsilon \oplus \epsilon\right) \longrightarrow \mathbb{C} P^{1}, \text { where } \gamma_{1}^{2} \equiv \gamma_{1} \otimes \gamma_{1} ; \\
& P\left(\eta_{(0, \alpha)}\right) \longrightarrow \mathbb{C} P^{2} \quad \text { for } \quad \alpha \in \mathbb{Z} ; \\
& P\left(\eta_{(1, \beta)}\right) \longrightarrow \mathbb{C} P^{2} \quad \text { for } \quad \beta \in \mathbb{Z} .
\end{aligned}
$$

Proof. Take $M \in \mathcal{C} \mathcal{P} \mathcal{M}_{2}^{6}$. Then the first stage $C_{1}$ of $M$ is either $\mathbb{C} P^{1}$ or $\mathbb{C} P^{2}$. We treat these two cases separately below.

CASE I: $C_{1}=\mathbb{C} P^{1}$. Note that any complex vector bundles over $\mathbb{C} P^{1}$ decomposes into a Whitney sum of line bundles. Therefore a $\mathbb{C} P$-manifold $M \in \mathcal{C P} \mathcal{M}_{2}^{6}$ with $C_{1}=\mathbb{C} P^{1}$ is a twostage generalized Bott manifold. By using this fact, we have the following proposition (also see [CMS10, CPS]).

Proposition 3.2. Let $M \in \mathcal{G B} \mathcal{M}_{2}^{6} \subset \mathcal{C P} \mathcal{M}_{2}^{6}$ be a generalized Bot manifold with $C_{1}=\mathbb{C} P^{1}$. Then $M$ is diffeomorphic to one of the following three distinct manifolds:

$$
\begin{aligned}
& P\left(\gamma_{1}^{0} \oplus \epsilon \oplus \epsilon\right) \cong \mathbb{C} P^{1} \times \mathbb{C} P^{2}, \text { where } \gamma_{1}^{0} \equiv \epsilon ; \\
& P\left(\gamma_{1} \oplus \epsilon \oplus \epsilon\right) ; \\
& P\left(\gamma_{1}^{2} \oplus \epsilon \oplus \epsilon\right) .
\end{aligned}
$$

Proof. Because all complex vector bundles over $\mathbb{C} P^{1}$ can be classified by 1 st Chern classes, together with the fact that any complex vector bundles over $\mathbb{C} P^{1}$ decomposes into a Whitney sum of line bundles, a complex rank 3 vector bundle $\eta$ splits into

$$
\eta \equiv \gamma_{1}^{k} \oplus \epsilon \oplus \epsilon
$$

where $c_{1}(\eta)=k c_{1}\left(\gamma_{1}\right)$. Moreover, by Lemma 2.1,

$$
\begin{aligned}
P\left(\gamma_{1}^{3} \oplus \epsilon \oplus \epsilon\right) & \cong P\left(\left(\gamma_{1}^{3} \oplus \epsilon \oplus \epsilon\right) \otimes \gamma_{1}^{-1}\right) \\
& \cong P\left(\gamma_{1}^{2} \oplus \gamma_{1}^{-1} \oplus \gamma_{1}^{-1}\right) .
\end{aligned}
$$

Because $c_{1}\left(\gamma_{1}^{2} \oplus \gamma_{1}^{-1} \oplus \gamma_{1}^{-1}\right)=0$, we have that

$$
P\left(\gamma_{1}^{3} \oplus \epsilon \oplus \epsilon\right) \cong \mathbb{C} P^{1} \times \mathbb{C} P^{2} .
$$

Comparing the cohomology rings of $P\left(\gamma_{1}^{1} \oplus \epsilon \oplus \epsilon\right)$ and $P\left(\gamma_{1}^{2} \oplus \epsilon \oplus \epsilon\right)$, we establish the statement. 
CASE II: $C_{1}=\mathbb{C} P^{2}$. Because $\operatorname{dim} M=6$ and $C_{1}=\mathbb{C} P^{2}$, the bundle $E_{1} \rightarrow C_{1}$ is a complex 2-dimensional vector bundle. Such vector bundles are determined by their Chern classes $c_{1}$ and $c_{2}$ (see $[\mathbf{S h}])$. Hence, by Lemmas 2.1 and 2.2 , we may denote $E_{1}$ by $\eta_{(s, \alpha)}$ such that $c_{1}\left(\eta_{(s, \alpha)}\right)=s x$ for $s=0,1$ and $c_{2}\left(\eta_{(s, \alpha)}\right)=\alpha x^{2} \in H^{4}\left(\mathbb{C} P^{2}\right)$ for $\alpha \in \mathbb{Z}$. In Case II, we have the following classification result.

Proposition 3.3. The following are equivalent for $s_{1}, s_{2} \in\{0,1\}$ and $\alpha_{1}, \alpha_{2} \in \mathbb{Z}$.

(1) $\left(s_{1}, \alpha_{1}\right)=\left(s_{2}, \alpha_{2}\right)$.

(2) Two manifolds $P\left(\eta_{\left(s_{1}, \alpha_{1}\right)}\right)$ and $P\left(\eta_{\left(s_{2}, \alpha_{2}\right)}\right)$ are diffeomorphic.

(3) Two cohomology rings $H^{*}\left(P\left(\eta_{\left(s_{1}, \alpha_{1}\right)}\right)\right)$ and $H^{*}\left(P\left(\eta_{\left(s_{2}, \alpha_{2}\right)}\right)\right)$ isomorphic.

Theorem 3.1 follows from Proposition 3.2 and 3.3.

It remains to prove Proposition 3.3.

Proof of Proposition 3.3. (1) $\Rightarrow(2)$ and $(2) \Rightarrow(3)$ are obvious. It is enough to show $(3) \Rightarrow(1)$. We prove this by proving the three claims: (1) $H^{*}\left(P\left(\eta_{(0, \alpha)}\right)\right) \not H^{*}\left(P\left(\eta_{(1, \beta)}\right)\right)$ for every $\alpha, \beta \in \mathbb{Z}$, (2) if $H^{*}\left(P\left(\eta_{\left(0, \alpha_{1}\right)}\right)\right) \simeq H^{*}\left(P\left(\eta_{\left(0, \alpha_{2}\right)}\right)\right)$ then $\alpha_{1}=\alpha_{2}$, and (3) if $H^{*}\left(P\left(\eta_{\left(1, \beta_{1}\right)}\right)\right) \simeq$ $H^{*}\left(P\left(\eta_{\left(1, \beta_{2}\right)}\right)\right)$ then $\beta_{1}=\beta_{2}$.

Claim 1: $H^{*}\left(P\left(\eta_{(0, \alpha)}\right)\right) \not 千 H^{*}\left(P\left(\eta_{(1, \beta)}\right)\right)$ for every $\alpha, \beta \in \mathbb{Z}$. By using the Borel-Hirzebruch formula (2.1), we have the following isomorphisms:

$$
\begin{aligned}
& H^{*}\left(P\left(\eta_{(0, \alpha)}\right)\right) \simeq \mathbb{Z}[X, Y] /\left\langle X^{3}, Y^{2}+\alpha X^{2}\right\rangle ; \\
& H^{*}\left(P\left(\eta_{(1, \beta)}\right)\right) \simeq \mathbb{Z}[x, y] /\left\langle x^{3}, y^{2}+x y+\beta x^{2}\right\rangle,
\end{aligned}
$$

where $\operatorname{deg} X=\operatorname{deg} Y=\operatorname{deg} x=\operatorname{deg} y=2$. We write the $\mathbb{Z}$-module structures of $H^{*}\left(P\left(\eta_{(0, \alpha)}\right)\right)$ and $H^{*}\left(P\left(\eta_{(1, \beta)}\right)\right)$ by indicating their generators as follows:

$$
\begin{aligned}
& \mathbb{Z} \oplus \mathbb{Z} X \oplus \mathbb{Z} Y \oplus \mathbb{Z} X^{2} \oplus \mathbb{Z} X Y \oplus \mathbb{Z} X^{2} Y ; \\
& \mathbb{Z} \oplus \mathbb{Z} x \oplus \mathbb{Z} y \oplus \mathbb{Z} x^{2} \oplus \mathbb{Z} x y \oplus \mathbb{Z} x^{2} y .
\end{aligned}
$$

If there exits a graded ring isomorphism $f: H^{*}\left(P\left(\eta_{(0, \alpha)}\right)\right) \rightarrow H^{*}\left(P\left(\eta_{(1, \beta)}\right)\right)$, then we may put $f(X)=a x+b y$ and $f(Y)=c x+d y$ for some $a, b, c, d \in \mathbb{Z}$ such that

$$
a d-b c= \pm 1 \text {. }
$$

Because $f$ preserves the ring structure, we have

$$
\begin{aligned}
f\left(X^{3}\right) & =(a x+b y)^{3} \\
& =\left(3 a^{2} b-3 a b^{2}+b^{3}-\beta b^{3}\right) x^{2} y=0 ; \\
f\left(Y^{2}+\alpha X^{2}\right) & =(c x+d y)^{2}+\alpha(a x+b y)^{2} \\
& =\left(c^{2}+\alpha a^{2}-\beta d^{2}-\alpha \beta b^{2}\right) x^{2}+\left(2 c d+2 \alpha a b-d^{2}-\alpha b^{2}\right) x y=0 .
\end{aligned}
$$

This implies the following equations:

$$
\begin{aligned}
& b\left(3 a^{2}-3 a b+b^{2}-\beta b^{2}\right)=0 \\
& c^{2}+\alpha a^{2}-\beta d^{2}-\alpha \beta b^{2}=0 \\
& 2 c d+2 \alpha a b-d^{2}-\alpha b^{2}=0
\end{aligned}
$$

If $b=0$, then $2 c=d= \pm 1$ by (3.1) and (3.4). But this contradicts to the fact that $c$ is an integer (i.e., $c \in \mathbb{Z}$ ). Hence $b \neq 0$, and by $(3.2)$ we have $3 a^{2}-3 a b+b^{2}-\beta b^{2}=0$. We also have the following commutative diagram of free $\mathbb{Z}$-modules.

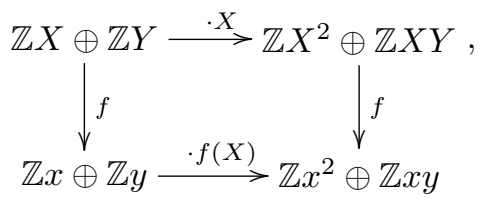


where the horizontal maps are induced from the multiplication by $X$ and $f(X)$, respectively. Let us represent the linear map $\cdot f(X)=\cdot(a x+b y): \mathbb{Z} x \oplus \mathbb{Z} y \rightarrow \mathbb{Z} x^{2} \oplus \mathbb{Z} x y$ by the matrix

$$
A=\left(\begin{array}{cc}
a & -\beta b \\
b & a-b
\end{array}\right)
$$

with respect to the generators. Note that $X: X X \oplus \mathbb{Z} Y \rightarrow \mathbb{Z} X^{2} \oplus \mathbb{Z} X Y$ is an isomorphism. Therefore $\cdot f(X)$ is also an isomorphism, and hence

$$
\operatorname{det} A=a^{2}-a b+\beta b^{2}= \pm 1 \text {. }
$$

Because $b \neq 0$, it follows from (3.2) and (3.5) that we have $b= \pm 1, \beta=1$ and $a=0$ or $b$. If $a=b$, then $c=d$ or $c=-d$ by (3.3). However, it is easy to check that both of these cases give contradictions to (3.1) and $c, d \in \mathbb{Z}$. Hence, $a=0$. In this case, $\alpha=c^{2}-d^{2}$ by (3.3) and $\alpha=2 c d-d^{2}$ by (3.4). Therefore we have $c=0$ or $2 d$. However, both of these cases give contradictions to $(3.1)$ and $c, d \in \mathbb{Z}$. This establishes that there is no ring isomorphism between $H^{*}\left(P\left(\eta_{(0, \alpha)}\right)\right)$ and $H^{*}\left(P\left(\eta_{(1, \beta)}\right)\right)$.

Claim 2: If $H^{*}\left(P\left(\eta_{\left(0, \alpha_{1}\right)}\right)\right) \simeq H^{*}\left(P\left(\eta_{\left(0, \alpha_{2}\right)}\right)\right)$, then $\alpha_{1}=\alpha_{2}$. By (2.1), we have the isomorphisms

$$
\begin{aligned}
& H^{*}\left(P\left(\eta_{\left(0, \alpha_{1}\right)}\right)\right) \simeq \mathbb{Z}[X, Y] /\left\langle X^{3}, Y^{2}+\alpha_{1} X^{2}\right\rangle, \text { and } \\
& H^{*}\left(P\left(\eta_{\left(0, \alpha_{2}\right)}\right)\right) \simeq \mathbb{Z}[x, y] /\left\langle x^{3}, y^{2}+\alpha_{2} x^{2}\right\rangle .
\end{aligned}
$$

Assume that there exists an isomorphism $f: H^{*}\left(P\left(\eta_{\left(0, \alpha_{1}\right)}\right)\right) \rightarrow H^{*}\left(P\left(\eta_{\left(0, \alpha_{2}\right)}\right)\right)$ for some $\alpha_{1}, \alpha_{2} \in$ $\mathbb{Z}$, and let $f(X)=a x+b y$ and $f(Y)=c x+d y$, so that $a d-b c= \pm 1$. Because $f\left(X^{3}\right)=(a x+b y)^{3}=$ 0 , we have that

$$
b\left(3 a^{2}-b^{2} \alpha_{2}\right)=0 .
$$

Suppose $b \neq 0$. Then $3 a^{2}-b^{2} \alpha_{2}=0$. Because the map

$$
f: H^{6}\left(P\left(\eta_{\left(0, \alpha_{1}\right)}\right)\right)=\mathbb{Z} X^{2} Y \longrightarrow \mathbb{Z} x^{2} y=H^{6}\left(P\left(\eta_{\left(0, \alpha_{2}\right)}\right)\right),
$$

is an isomorphism, we have

$$
f\left(X^{2} Y\right)=(a x+b y)^{2}(c x+d y)= \pm x^{2} y .
$$

Using (3.6) and the ring structures, we have that

$$
a^{2} d+2 a b c-b^{2} d \alpha_{2}= \pm 1 \text {. }
$$

Because $3 a^{2}-b^{2} \alpha_{2}=0$, we have $-2 a^{2} d+2 a b c=-2 a(a d-b c)= \pm 1$. However, this gives a contradiction to $a \in \mathbb{Z}$, because $a d-b c= \pm 1$. Hence, $b=0$ and $a d= \pm 1$; in particular, we have $a, d= \pm 1$. Then, we have the following equations:

$$
\begin{aligned}
f\left(Y^{2}+\alpha_{1} X^{2}\right) & =(c x+d y)^{2}+\alpha_{1}(a x+b y)^{2} \\
& =\left(c^{2}-\alpha_{2}+\alpha_{1}\right) x^{2}+2 c d x y=0 .
\end{aligned}
$$

Therefore, we have that $c=0$ and $\alpha_{1}=\alpha_{2}$. This proves the claim.

Claim 3: If $H^{*}\left(P\left(\eta_{\left(1, \beta_{1}\right)}\right)\right) \simeq H^{*}\left(P\left(\eta_{\left(1, \beta_{2}\right)}\right)\right)$, then $\beta_{1}=\beta_{2}$. By $(2.1)$, we have the isomorphisms

$$
\begin{aligned}
& H^{*}\left(P\left(\eta_{\left(1, \beta_{1}\right)}\right)\right) \simeq \mathbb{Z}[X, Y] /\left\langle X^{3}, Y^{2}+X Y+\beta_{1} X^{2}\right\rangle, \text { and } \\
& H^{*}\left(P\left(\eta_{\left(1, \beta_{2}\right)}\right)\right) \simeq \mathbb{Z}[x, y] /\left\langle x^{3}, y^{2}+x y+\beta_{2} x^{2}\right\rangle .
\end{aligned}
$$

Assume that there exists an isomorphism $f: H^{*}\left(P\left(\eta_{\left(1, \beta_{1}\right)}\right)\right) \rightarrow H^{*}\left(P\left(\eta_{\left(1, \beta_{2}\right)}\right)\right)$ for some $\beta_{1}, \beta_{2} \in \mathbb{Z}$, and let $f(X)=a x+b y$ and $f(Y)=c x+d y$, so that $a d-b c= \pm 1$. Because of the relations $f\left(X^{3}\right)=(a x+b y)^{3}=0$ and $f\left(Y^{2}+X Y+\beta_{1} X^{2}\right)=(c x+d y)^{2}+(a x+b y)(c x+d y)+\beta_{1}(a x+b y)^{2}=0$, we have that

$$
\begin{gathered}
b\left(3 a^{2}-3 a b+b^{2}-b^{2} \beta_{2}\right)=0 \\
c^{2}-d^{2} \beta_{2}+a c-b d \beta_{2}+a^{2} \beta_{1}-b^{2} \beta_{1} \beta_{2}=0 \\
2 c d-d^{2}+a d+b c-b d+2 \beta_{1} a b-\beta_{1} b^{2}=0 .
\end{gathered}
$$


We first assume $b=0$. From the equation $a d-b c= \pm 1$, we have $a, d= \pm 1$. Now plug $b=0$ and $d= \pm 1$ into (3.9) to get the equation

$$
2 c+a=d= \pm 1 .
$$

Together with $a= \pm 1$, this equation implies that either $c=0$ and $a=d$, or $c \neq 0$ and $c=-a=d$. Now plug these into (3.8) to obtain $\beta_{1}=\beta_{2}$ in either cases, which proves the claim when $b=0$.

We now assume $b \neq 0$. Then from (3.7), we have $3 a^{2}-3 a b+b^{2}-b^{2} \beta_{2}=0$. By using the same argument as the one used to get (3.5), we have

$$
a^{2}-a b+\beta_{2} b^{2}=\epsilon,
$$

where $\epsilon= \pm 1$. Substitute (3.10) into the equation $3 a^{2}-3 a b+b^{2}-b^{2} \beta_{2}=0$. Then, we obtain the equation

$$
b^{2}\left(4 \beta_{2}-1\right)=3 \epsilon .
$$

Therefore, $b= \pm 1$ and $\beta_{2}=\epsilon=1$. Hence, together with (3.10), we have that $a=0$ or $a=b$.

If $a=0$, then $c= \pm 1$ by the equation $a d-b c= \pm 1$. Substitute these equations into (3.8) and (3.9). Then, we have the equations

$$
\beta_{1}=1-d^{2}-b d=2 c d-d^{2}+b c-b d .
$$

Therefore, we have that $(2 d+b) c=1$. Moreover, because $c= \pm 1$ and $b= \pm 1$, we have $(b, d)=(c, 0)$ or $(-c, c)$. Hence, $\beta_{1}=1=\beta_{2}$.

If $a=b= \pm 1$, then $d-c= \pm 1$ by the equation $a d-b c= \pm 1$. Put $a=b= \pm 1$ in (3.9) to obtain the equation

$$
\beta_{1}=d^{2}-2 c d-b c .
$$

Moreover, by substituting $a=b= \pm 1$ and $\beta_{2}=1$ into (3.8), we have

$$
(c-d)(a+c+d)=0 .
$$

This together with $d-c= \pm 1$ implies that $c+d=-a= \pm 1$. It follows that either $d=0$ and $c=-a=-b$, or $d=-a=-b$ and $c=0$. By (3.11), we have $\beta_{1}=1=\beta_{2}$. This proves the claim, and hence the proof of the proposition is complete.

We can show easily that $P\left(\eta_{(s, \alpha)}\right)$ is diffeomorphic to $\mathbb{C} P^{1} \times \mathbb{C} P^{2}$ if and only if $(s, \alpha)=(0,0)$ by comparing their cohomology rings. Therefore, by Propositions 3.2 and 3.3, we have Theorem 3.1. Moreover, by Theorem 3.1, we have the following corollary.

Corollary 3.4. Let $\mathcal{C P} \mathcal{M}_{\leq 2}^{6}$ be the class of all 6 -dimensional $\mathbb{C} P$-manifolds of height at most 2. Then, two $\mathbb{C} P$-manifolds $M$ and $M^{\prime}$ in $\mathcal{C P} \mathcal{M}_{\leq 2}^{6}$ are diffeomorphic if and only if their cohomology rings $H^{*}(M)$ and $H^{*}\left(M^{\prime}\right)$ are isomorphic. In other words, the class $\mathcal{C P} \mathcal{M}_{\leq 2}^{6}$ is cohomologically rigid.

\section{The class $\mathcal{C P} \mathcal{M}_{3}^{6}$}

In this final section, we focus on $\mathcal{C P} \mathcal{M}_{3}^{6}$, i.e., the class of 6 -dimensional $\mathbb{C} P$-manifolds of height 3. The elements in $\mathcal{C P} \mathcal{M}_{3}^{6}$ are of the form

$$
P(\xi) \stackrel{\mathbb{C} P^{1}}{\longrightarrow} H_{k} \stackrel{\mathbb{C} P^{1}}{\longrightarrow} \mathbb{C} P^{1} .
$$

Here, $\xi$ is a complex 2-dimensional vector bundle over $H_{k}$, and $H_{k}$ is the Hirzebruch surface $P\left(\gamma_{1}^{k} \oplus \epsilon\right)$ where $\epsilon$ is the trivial complex line bundle and $\gamma_{1}^{k}$ is the $k$-th tensor power of the tautological line bundle $\gamma_{1}$ over $\mathbb{C} P^{1}$. As is well known, $H_{k}$ is diffeomorphic to $H_{0}$ if $k$ is even, and to $H_{1}$ if $k$ is odd (see $[\mathbf{H i}, \mathbf{M a S u}]$ ).

LEMMA 4.1. Let $\operatorname{Vect}_{\mathbb{C}}^{2}\left(H_{k}\right)$ be the set of complex 2-dimensional vector bundles over $H_{k}$ up to isomorphisms. Then the correspondence

$$
\begin{array}{ccc}
\operatorname{Vect}_{\mathbb{C}}^{2}\left(H_{k}\right) & \stackrel{c}{\longrightarrow} & H^{2}\left(H_{k}\right) \oplus H^{4}\left(H_{k}\right) \\
\stackrel{\Psi}{\xi} & \longmapsto & c_{1}(\xi) \oplus c_{2}(\xi)
\end{array}
$$


is bijective.

Proof. Since $\operatorname{dim}_{\mathbb{R}} H_{k}=4$, any two bundles $\eta_{1}$ and $\eta_{2} \in \operatorname{Vect}_{\mathbb{C}}^{2}\left(H_{k}\right)$ are isomorphic if and only if they are stably isomorphic, i.e., $\eta_{1} \oplus \epsilon^{\ell} \equiv \eta_{2} \oplus \epsilon^{\ell}$ for some trivial complex $\ell$-dimensional bundle $\epsilon^{\ell}$, see [Hu, 1.5 Theorem in Chapter 9]. Therefore $\eta_{1}$ and $\eta_{2}$ represent the same element in $\widetilde{K}\left(H_{k}\right)$, the stable K-ring of $H_{k}$, if and only if $\eta_{1} \equiv \eta_{2}$. Therefore the map $\operatorname{Vect}_{\mathbb{C}}^{2}\left(H_{k}\right) \rightarrow \widetilde{K}\left(H_{k}\right)$ defined by $\xi \mapsto[\xi]$ is bijective. Hence, it is enough to prove that the induced map

$$
c^{\prime}: \widetilde{K}\left(H_{k}\right) \rightarrow H^{2}\left(H_{k}\right) \oplus H^{4}\left(H_{k}\right), \quad[\xi] \mapsto\left(c_{1}(\xi), c_{2}(\xi)\right)
$$

is bijective.

Let $s: \mathbb{C} P^{1} \rightarrow H_{k}=P\left(\gamma_{1}^{k} \oplus \epsilon^{1}\right)$ be the section defined by $s([p])=[p,[0: 1]]$, and let $i: \mathbb{C} P^{1} \rightarrow$ $H_{k}$ be an inclusion to a fiber in the fibration $H_{k} \rightarrow \mathbb{C} P^{1}$. Then $s\left(\mathbb{C} P^{1}\right) \cup i\left(\mathbb{C} P^{1}\right) \cong \mathbb{C} P^{1} \vee \mathbb{C} P^{1}$, and we have the following inclusion and collapsing sequence

$$
\mathbb{C} P^{1} \vee \mathbb{C} P^{1} \longrightarrow H_{k} \longrightarrow H_{k} /\left(\mathbb{C} P^{1} \vee \mathbb{C} P^{1}\right)
$$

Since $H_{k}$ admits a CW-structure with one 0-cell, two 2-cells, and one 4-cell (e.g. see [DaJa]), $H_{k} /\left(\mathbb{C} P^{1} \vee \mathbb{C} P^{1}\right)$ may be regarded as the collapsing of two 2-cells to the one 0-cell. Therefore, the space $H_{k} /\left(\mathbb{C} P^{1} \vee \mathbb{C} P^{1}\right)$ is homeomorphic to $S^{4}$. Hence, we have the following exact sequence of reduced $K$ groups (see $[\mathbf{H u}, 2.1$ Proposition in Chapter 10]):

$$
\widetilde{K}\left(S^{4}\right) \rightarrow \widetilde{K}\left(H_{k}\right) \rightarrow \widetilde{K}\left(\mathbb{C} P^{1} \vee \mathbb{C} P^{1}\right)
$$

As is well known, we have the following isomorphisms

$$
\begin{aligned}
& \widetilde{K}\left(S^{4}\right) \simeq \widetilde{K}\left(S^{2}\right) \simeq \widetilde{K}\left(\mathbb{C} P^{1}\right) \simeq \mathbb{Z}, \text { and } \\
& \widetilde{K}\left(\mathbb{C} P^{1} \vee \mathbb{C} P^{1}\right) \simeq \widetilde{K}\left(\mathbb{C} P^{1}\right) \oplus \widetilde{K}\left(\mathbb{C} P^{1}\right) \simeq \mathbb{Z} \oplus \mathbb{Z}=\mathbb{Z}^{2} .
\end{aligned}
$$

These isomorphisms are induced by taking the Chern classes of vector bundles. Let $c^{\prime}=\left(c_{1}^{\prime}, c_{2}^{\prime}\right)$ : $\widetilde{K}\left(H_{k}\right) \rightarrow H^{2}\left(H_{k}\right) \oplus H^{4}\left(H_{k}\right) \simeq \mathbb{Z}^{2} \oplus \mathbb{Z}$, where $c_{1}^{\prime}([\xi])=c_{1}(\xi)$ and $c_{2}^{\prime}([\xi])=c_{2}(\xi)$. Then $c_{1}^{\prime}$ : $\widetilde{K}\left(H_{k}\right) \rightarrow H^{2}\left(H_{k}\right)$ is surjective because for any $\alpha \in H^{2}\left(H_{k}\right) \simeq \mathbb{Z}^{2}$ can be realized as the first Chern class $c_{1}(\gamma)$ of a complex line bundle $\gamma$ over $H_{k}$. Indeed, for a given $\alpha_{1} x+\alpha_{2} y \in \mathbb{Z} x \oplus \mathbb{Z} y=H^{2}\left(H_{k}\right)$, the line bundle $\gamma=\pi^{*}\left(\gamma_{1}^{\alpha_{1}}\right) \otimes \gamma_{H_{k}}^{\alpha_{2}}$ has the first Chern class $\alpha_{1} x+\alpha_{2} y$, where $\pi: H_{k} \rightarrow \mathbb{C} P^{1}$ is the projection, $\gamma_{H_{k}}$ is the canonical line bundle over $H_{k}=P\left(\gamma_{1}^{k} \oplus \epsilon^{1}\right)$ induced from the vector bundle $\pi^{*}\left(\gamma_{\tilde{\sigma}}^{k} \oplus \epsilon^{1}\right)$, and $x, y$ are generators induced by $c_{1}\left(\pi^{*} \gamma_{1}\right), c_{1}\left(\gamma_{H_{k}}\right)$ respectively. We also claim that $c_{2}^{\prime}: \widetilde{K}\left(H_{k}\right) \rightarrow H^{4}\left(H_{k}\right)$ is surjective. By the fundamental results of fibre bundle, we can construct all complex 2-dimensional vector bundles over $H_{k} /\left(\mathbb{C} P^{1} \vee \mathbb{C} P^{1}\right) \cong S^{4}$ by using the continuous map $S^{4} \rightarrow B U(2)$ up to homotopy. Because $\pi_{4}(B U(2)) \simeq \mathbb{Z}$, for a given $\beta \in H^{4}\left(H_{k} /\left(\mathbb{C} P^{1} \vee \mathbb{C} P^{1}\right)\right)$ we can construct the complex 2-dimensional vector bundle $\eta^{\prime}$ such that $c\left(\eta^{\prime}\right)=1+\beta$. Now the collapsing map $\rho: H_{k} \rightarrow H_{k} /\left(\mathbb{C} P^{1} \vee \mathbb{C} P^{1}\right)$ induces the isomorphism $H^{4}\left(H_{k} /\left(\mathbb{C} P^{1} \vee \mathbb{C} P^{1}\right)\right) \simeq$ $H^{4}\left(H_{k}\right) \simeq \mathbb{Z}$; therefore, its pull-back $\eta=\rho^{*} \eta^{\prime}$ over $H_{k}$ satisfies $c(\eta)=1+\beta$. This implies that $c_{2}^{\prime}$ is surjective. Because $\gamma \oplus \eta$ is a complex 3 -dimensional vector bundle and $\operatorname{dim}_{\mathbb{R}} H_{k}=4$, the bundle $\gamma \oplus \eta$ is in the stable range. Therefore, there is the complex 2-dimensional vector bundle $\xi$ such that $\xi \oplus \epsilon^{1} \equiv \gamma \oplus \eta$, where $\epsilon^{1}$ is the trivial line bundle over $H_{k}$, and $c(\xi)=c(\gamma \oplus \eta)=1+c_{1}(\gamma)+c_{2}(\eta)$. Therefore, the map $c^{\prime}: \widetilde{K}\left(H_{k}\right) \rightarrow H^{2}\left(H_{k}\right) \oplus H^{4}\left(H_{k}\right)$ is surjective. Now consider the following diagram.

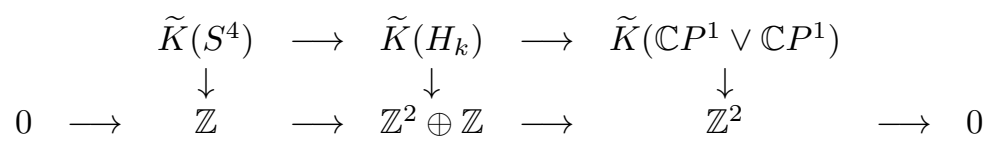

Here the vertical maps from the left are the isomorphism in $(4.1)$, the map $c^{\prime}: \widetilde{K}\left(H_{k}\right) \rightarrow H^{2}\left(H_{k}\right) \oplus$ $H^{4}\left(H_{k}\right)$ and the isomorphism in (4.2), and the horizontal sequences are exact. One can see easily that the diagram is commutative. From the commutativity of the diagram and the surjectivity of the map $c^{\prime}$, we can see that $\widetilde{K}\left(S^{4}\right) \rightarrow \widetilde{K}\left(H_{k}\right) \rightarrow \widetilde{K}\left(\mathbb{C} P^{1} \vee \mathbb{C} P^{1}\right)$ is a short exact sequence, and the map $c^{\prime}$ is bijective. Consequently, there exists the bijective map $\operatorname{Vect}_{\mathbb{C}}^{2}\left(H_{k}\right) \rightarrow H^{2}\left(H_{k}\right) \oplus H^{4}\left(H_{k}\right)$ defined by $\xi \mapsto c_{1}(\xi) \oplus c_{2}(\xi)$. This establishes the lemma. 
By Lemma 4.1, any complex 2-dimensional vector bundles over $H_{0}$ and $H_{1}$ can be written by

$$
\eta_{(s, r, \alpha)} \rightarrow H_{0}, \text { and } \xi_{(s, r, \beta)} \rightarrow H_{1}
$$

where

$$
\begin{gathered}
c_{1}\left(\eta_{(s, r, \alpha)}\right)=(s, r) \in H^{2}\left(H_{0}\right) \simeq \mathbb{Z} \oplus \mathbb{Z}, \quad c_{2}\left(\eta_{(s, r, \alpha)}\right)=\alpha \in H^{4}\left(H_{0}\right) \simeq \mathbb{Z} ; \\
c_{1}\left(\xi_{(s, r, \beta)}\right)=(s, r) \in H^{2}\left(H_{1}\right) \simeq \mathbb{Z} \oplus \mathbb{Z}, \quad c_{2}\left(\xi_{(s, r, \beta)}\right)=\beta \in H^{4}\left(H_{1}\right) \simeq \mathbb{Z} .
\end{gathered}
$$

Moreover, by taking tensor product with an appropriate line bundle if necessary, we may assume $(s, r) \in\{0,1\}^{2}$, see Lemma 2.2. The main theorem of this section is the following.

THEOREM 4.2. The element in $\mathcal{C} \mathcal{P} \mathcal{M}_{3}^{6}$ consists of the following distinct manifolds:

$$
\begin{aligned}
& P\left(\eta_{(0,0, \alpha)}\right) \text { for } \alpha \in \mathbb{Z}_{\geq 0} ; \\
& P\left(\eta_{(1,0, \alpha)}\right) \text { for } \alpha \in \mathbb{Z}_{\geq 0} ; \\
& P\left(\eta_{(1,1, \alpha)}\right) \text { for } \alpha \in \mathbb{N} ; \\
& P\left(\xi_{(0,0, \beta)}\right) \text { for } \beta \in \mathbb{N} ; \\
& P\left(\xi_{(1,0, \beta)}\right) \text { for } \beta \in \mathbb{Z}_{\geq 0} ; \\
& P\left(\xi_{(0,1, \beta)}\right) \text { for } \beta \in \mathbb{Z} .
\end{aligned}
$$

Moreover, we have the diffeomorphisms $P\left(\eta_{(1,0, \alpha)}\right) \cong P\left(\eta_{(0,1, \alpha)}\right), P\left(\eta_{(1,0,0)}\right) \cong P\left(\xi_{(0,0,0)}\right)=\mathbb{C} P^{1} \times$ $H_{1}$, and $P\left(\xi_{(0,1, \beta)}\right) \cong P\left(\xi_{(1,1,-\beta)}\right)$.

To prove Theorem 4.2, we first observe the following. For $H_{0}=\mathbb{C} P^{1} \times \mathbb{C} P^{1}$, there is a selfdiffeomorphism on $H_{0}$ defined by exchanging the first and second terms, i.e., $(p, q) \mapsto(q, p)$ for $(p, q) \in H_{0}=\mathbb{C} P^{1} \times \mathbb{C} P^{1}$. This diffeomorphism induces a bundle isomorphism between $\eta_{(s, r, \alpha)}$ and $\eta_{(r, s, \alpha)}$. Therefore, we may assume $(s, r)=(0,0),(1,0)$ or $(1,1)$ in the case of $\eta_{(s, r, \alpha)}$.

We also need the following lemma.

LEMmA 4.3. If the cohomology ring $H^{*}\left(P\left(\eta_{(s, r, \alpha)}\right)\right)$ is isomorphic to $H^{*}\left(P\left(\xi_{\left(s^{\prime}, r^{\prime}, \beta\right)}\right)\right)$, then $(s, r, \alpha)=(1,0,0)$ and $\left(s^{\prime}, r^{\prime}, \beta\right)=(0,0,0)$. Furthermore, $P\left(\eta_{(1,0,0)}\right)$ is diffeomorphic to $P\left(\xi_{(0,0,0)}\right)$.

Proof. By the Borel Hirzebruch formula (2.1), we have the isomorphisms

$$
\begin{aligned}
H^{*}\left(P\left(\eta_{(s, r, \alpha)}\right)\right) & \simeq \mathbb{Z}[X, Y, Z] /\left\langle X^{2}, Y^{2}, Z^{2}+s Z X+r Z Y+\alpha X Y\right\rangle, \text { and } \\
H^{*}\left(P\left(\xi_{\left(s^{\prime}, r^{\prime}, \beta\right)}\right)\right) & \simeq \mathbb{Z}[x, y, z] /\left\langle x^{2}, y^{2}+x y, z^{2}+s^{\prime} z x+r^{\prime} z y+\beta x y\right\rangle,
\end{aligned}
$$

where $(s, r)=(0,0),(1,0)$ or $(1,1)$ in $\eta_{(s, r, \alpha)}$, and $\left(s^{\prime}, r^{\prime}\right)=(0,0),(1,0),(0,1)$ or $(1,1)$ in $\xi_{\left(s^{\prime}, r^{\prime}, \alpha\right)}$. For each $(s, r, \alpha)$ and $\left(s^{\prime}, r^{\prime}, \beta\right)$, we express the $\mathbb{Z}$-module structures of the above cohomology rings using their generators as follows:

\section{$\mathbb{Z} \oplus \mathbb{Z} X \oplus \mathbb{Z} Y \oplus \mathbb{Z} Z \oplus \mathbb{Z} X Y \oplus \mathbb{Z} Y Z \oplus \mathbb{Z} Z X \oplus \mathbb{Z} X Y Z$ \\ $\mathbb{Z} \oplus \mathbb{Z} x \oplus \mathbb{Z} y \oplus \mathbb{Z} z \oplus \mathbb{Z} x y \oplus \mathbb{Z} y z \oplus \mathbb{Z} z x \oplus \mathbb{Z} x y z$.}

Assume there exists an isomorphism $f: H^{*}\left(P\left(\eta_{(s, r, \alpha)}\right)\right) \rightarrow H^{*}\left(P\left(\xi_{\left(s^{\prime}, r^{\prime}, \beta\right)}\right)\right)$. Let $f(X)=$ $a_{1} x+b_{1} y+c_{1} z, f(Y)=a_{2} x+b_{2} y+c_{2} z$ and $f(Z)=a_{3} x+b_{3} y+c_{3} z$, and let $A_{f}$ denote the corresponding $3 \times 3$ matrix of $f$. Because $f$ is a graded ring isomorphism, it satisfies the following relations:

$$
\begin{aligned}
& f(X)^{2}=\left(a_{1} x+b_{1} y+c_{1} z\right)^{2}=\left(2 a_{1} b_{1}-b_{1}^{2}-\beta c_{1}^{2}\right) x y+\left(2 a_{1} c_{1}-s^{\prime} c_{1}^{2}\right) x z+\left(2 b_{1} c_{1}-r^{\prime} c_{1}^{2}\right) y z=0 \\
& f(Y)^{2}=\left(a_{2} x+b_{2} y+c_{2} z\right)^{2}=\left(2 a_{2} b_{2}-b_{2}^{2}-\beta c_{2}^{2}\right) x y+\left(2 a_{2} c_{2}-s^{\prime} c_{2}^{2}\right) x z+\left(2 b_{2} c_{2}-r^{\prime} c_{2}^{2}\right) y z=0
\end{aligned}
$$

in $H^{*}\left(P\left(\xi_{\left(s^{\prime}, r^{\prime}, \beta\right)}\right)\right)$. Therefore, we have

$$
\begin{aligned}
& 2 a_{i} b_{i}-b_{i}^{2}-\beta c_{i}^{2}=0 \\
& 2 a_{i} c_{i}-s^{\prime} c_{i}^{2}=0 ; \\
& 2 b_{i} c_{i}-r^{\prime} c_{i}^{2}=0,
\end{aligned}
$$

for $i=1,2$.

Assume $c_{1}=0$. Then, by using the first equation above and $\operatorname{det} A_{f}= \pm 1$, we have either $b_{1}=0$ and $a_{1}=\epsilon_{1}$, or $b_{1}=2 a_{1}=2 \epsilon_{1}$, where $\epsilon_{1}= \pm 1$. If $c_{2}=0$, then it is easy to check that this 
gives a contradiction to $\operatorname{det} A_{f}= \pm 1$. Hence, $c_{2} \neq 0$. By using the second and the third equations above, we have $s^{\prime} c_{2}=2 a_{2}$ and $r^{\prime} c_{2}=2 b_{2}$. Hence it can be seen easily from $\operatorname{det} A_{f}= \pm 1$ that only $\left(s^{\prime}, r^{\prime}\right)=(0,0)$ is possible, and in this case $\left(a_{2}, b_{2}, c_{2}\right)=\left(0,0, \epsilon_{2}\right)$ and $\beta=0$, where $\epsilon_{2}= \pm 1$. Hence, we have that $\left(s^{\prime}, r^{\prime}, \beta\right)=(0,0,0)$.

If $\left(a_{1}, b_{1}, c_{1}\right)=\left(\epsilon_{1}, 0,0\right)$, then $b_{3}=\epsilon_{3}$ because $\operatorname{det} A_{f}= \pm 1$. Therefore, it follows from $f(Z)^{2}=-s f(X) f(Z)-r f(Y) f(Z)-\alpha f(X) f(Y)$ that

$$
\begin{aligned}
& 2 a_{3} \epsilon_{3}-1=-s \epsilon_{1} \epsilon_{3} ; \\
& 2 a_{3} c_{3}=-s \epsilon_{1} c_{3}-r \epsilon_{2} a_{3}-\alpha \epsilon_{1} \epsilon_{2} \\
& 2 \epsilon_{3} c_{3}=-r \epsilon_{2} \epsilon_{3} .
\end{aligned}
$$

Using the third equation above, we have $r=c_{3}=0$. Therefore, by the second equation, we also have $\alpha=0$. Moreover, from the first equation $s=1$. Hence, $(s, r, \alpha)=(1,0,0)$.

If $\left(a_{1}, b_{1}, c_{1}\right)=\left(\epsilon_{1}, 2 \epsilon_{1}, 0\right)$, then $b_{3}-2 a_{3}=\epsilon_{3}$ because $\operatorname{det} A_{f}= \pm 1$. Therefore, it follows from $f(Z)^{2}=-s f(X) f(Z)-r f(Y) f(Z)-\alpha f(X) f(Y)$ that

$$
\begin{aligned}
& 2 a_{3} b_{3}-b_{3}^{2}=s \epsilon_{1} b_{3}-2 s \epsilon_{1} a_{3} ; \\
& 2 a_{3} c_{3}=-s \epsilon_{1} c_{3}-r \epsilon_{2} a_{3}-\alpha \epsilon_{1} \epsilon_{2} ; \\
& 2 b_{3} c_{3}=-r \epsilon_{2} b_{3}-2 s \epsilon_{1} c_{3}-2 \alpha \epsilon_{1} \epsilon_{2} .
\end{aligned}
$$

Using the first equation and $b_{3}-2 a_{3}=\epsilon_{3}$, we have $b_{3}=-s \epsilon_{1}$. Therefore, by using the third equation, we have $s r=-2 \alpha$. This implies that $\alpha=0$ and $s r=0$. If $s=0$, then $b_{3}=-s \epsilon_{1}=0$; however, $b_{3}-2 a_{3}=-2 a_{3}=\epsilon_{3}$ and this gives a contradiction. Therefore $(s, r, \alpha)=(1,0,0)$. This establishes the first statement of the lemma when $c_{1}=0$ case.

In the case when $c_{1} \neq 0$ and $c_{2}=0$, by a similar argument to the above case, we have the same result. When $c_{1} \neq 0$ and $c_{2} \neq 0$, by some routine computation, we can see that this case gives a contradiction. This establishes the first statement of the lemma.

Because $\eta_{(1,0,0)} \equiv \gamma_{x} \oplus \epsilon$, where $\gamma_{x}$ is the tautological line bundle along the first factor of $\mathbb{C} P^{1} \times \mathbb{C} P^{1}$, we can easily check that $P\left(\eta_{(1,0,0)}\right) \cong\left(S^{3} \times \mathbb{C} P^{1}\right) \times_{T^{1}} P\left(\mathbb{C}_{1} \oplus \mathbb{C}\right)$, where $T^{1}$ acts on $S^{3}$ as diagonal multiplications in its coordinates and trivially on $\mathbb{C} P^{1}$ and $\mathbb{C}_{1}$ is a complex 1-dimensional $T^{1}$ representation such that $t \cdot z=t z$ for $t \in T^{1}$ and $z \in \mathbb{C}_{1}$. On the other hand, because $\xi_{(0,0,0)}$ is the trivial bundle over $H_{1}$ (by Lemma 4.1), we have that $P\left(\xi_{(0,0,0)}\right)=S^{3} \times_{T^{1}} P\left(\mathbb{C}_{1} \oplus \mathbb{C}\right) \times \mathbb{C} P^{1}$. Therefore, we have that $P\left(\eta_{(1,0,0)}\right) \cong P\left(\xi_{(0,0,0)}\right)$. This establishes the second statement.

In order to prove Theorem 4.2, we may divide the proof into the following two cases.

CASE I: $P\left(\eta_{(s, r, \alpha)}\right)$ with the base space $H_{0}$. In this case $(s, r)=(0,0),(1,0)$ and $(1,1)$.

CASE II: $P\left(\xi_{(s, r, \alpha)}\right)$ with the base space $H_{1}$. In this case $(s, r)=(0,0),(1,0),(0,1)$ and $(1,1)$. Moreover if $(s, r)=(0,0)$ then $\alpha \neq 0$.

The rest of the section in devoted to the proof of Theorem 4.2 by treating the two cases separately.

CASE I: $P\left(\eta_{(s, r, \alpha)}\right)$ with the base space $H_{0}$. We prove the cohomological rigidity for $P\left(\eta_{(s, r, \alpha)}\right)$. Namely, we prove the following proposition.

Proposition 4.4. The following statements are equivalent.

(1) Two manifolds $P\left(\eta_{\left(s_{1}, r_{1}, \alpha_{1}\right)}\right)$ and $P\left(\eta_{\left(s_{2}, r_{2}, \alpha_{2}\right)}\right)$ are diffeomorphic.

(2) Two cohomology rings $H^{*}\left(P\left(\eta_{\left(s_{1}, r_{1}, \alpha_{1}\right)}\right)\right)$ and $H^{*}\left(P\left(\eta_{\left(s_{2}, r_{2}, \alpha_{2}\right)}\right)\right)$ are isomorphic.

(3) $\left(s_{1}, r_{1}\right)=\left(s_{2}, r_{2}\right)$, and $\alpha_{1}$ and $\alpha_{2}$ are as follows:

(a) if $\left(s_{1}, r_{1}\right)=\left(s_{2}, r_{2}\right)=(0,0)$, then $\alpha_{2}=\alpha_{1}$ or $-\alpha_{1}$;

(b) if $\left(s_{1}, r_{1}\right)=\left(s_{2}, r_{2}\right)=(1,0)$ (or $\left.(0,1)\right)$, then $\alpha_{2}=\alpha_{1}$ or $-\alpha_{1}$;

(c) if $\left(s_{1}, r_{1}\right)=\left(s_{2}, r_{2}\right)=(1,1)$, then $\alpha_{2}=\alpha_{1}$ or $-\alpha_{1}+1$.

Proof. $(1) \Rightarrow(2)$ is trivial.

We first prove $(2) \Rightarrow(3)$. By (2.1), we have the following isomorphisms

$$
\begin{aligned}
& H^{*}\left(P\left(\eta_{\left(s_{1}, r_{1}, \alpha_{1}\right)}\right)\right) \simeq \mathbb{Z}[X, Y, Z] /\left\langle X^{2}, Y^{2}, Z^{2}+s_{1} Z X+r_{1} Z Y+\alpha_{1} X Y\right\rangle, \text { and } \\
& H^{*}\left(P\left(\eta_{\left(s_{2}, r_{2}, \alpha_{2}\right)}\right)\right) \simeq \mathbb{Z}[x, y, z] /\left\langle x^{2}, y^{2}, z^{2}+s_{2} z x+r_{2} z y+\alpha_{2} x y\right\rangle .
\end{aligned}
$$


Assume there exists a graded ring isomorphism $f: H^{*}\left(P\left(\eta_{\left(s_{1}, r_{1}, \alpha_{1}\right)}\right)\right) \simeq H^{*}\left(P\left(\eta_{\left(s_{2}, r_{2}, \alpha_{2}\right)}\right)\right)$, and put the matrix representation of $f: H^{2}\left(P\left(\eta_{\left(s_{1}, r_{1}, \alpha_{1}\right)}\right)\right) \simeq H^{2}\left(P\left(\eta_{\left(s_{2}, r_{2}, \alpha_{2}\right)}\right)\right)$ with respect to the given module generators as

$$
A_{f}=\left(\begin{array}{lll}
a_{1} & b_{1} & c_{1} \\
a_{2} & b_{2} & c_{2} \\
a_{3} & b_{3} & c_{3}
\end{array}\right),
$$

i.e., $f(X)=a_{1} x+b_{1} y+c_{1} z, f(Y)=a_{2} x+b_{2} y+c_{2} z, f(Z)=a_{3} x+b_{3} y+c_{3} z$. Note that $\operatorname{det} A_{f}= \pm 1$. Because $X^{2}=Y^{2}=0$ and $f$ is a ring isomorphism,

$$
\begin{aligned}
& f(X)^{2}=\left(2 a_{1} b_{1}-\alpha_{2} c_{1}^{2}\right) x y+\left(2 a_{1}-s_{2} c_{1}\right) c_{1} x z+\left(2 b_{1}-r_{2} c_{1}\right) c_{1} y z=0 ; \\
& f(Y)^{2}=\left(2 a_{2} b_{2}-\alpha_{2} c_{2}^{2}\right) x y+\left(2 a_{2}-s_{2} c_{2}\right) c_{2} x z+\left(2 b_{2}-r_{2} c_{2}\right) c_{2} y z=0
\end{aligned}
$$

in $H^{*}\left(P\left(\eta_{\left(s_{2}, r_{2}, \alpha_{2}\right)}\right)\right)$. Therefore, we have

$$
\begin{aligned}
& 2 a_{i} b_{i}-\alpha_{2} c_{i}^{2}=0, \\
& \left(2 a_{i}-s_{2} c_{i}\right) c_{i}=0, \\
& \left(2 b_{i}-r_{2} c_{i}\right) c_{i}=0,
\end{aligned}
$$

for $i=1,2$. We divide the proof into the following three cases: Case $\mathbf{1}\left(s_{2}, r_{2}\right)=(1,1)$; Case 2 $\left(s_{2}, r_{2}\right)=(0,0)$; Case $3\left(s_{2}, r_{2}\right)=(1,0)$.

Case 1: $\left(s_{2}, r_{2}\right)=(1,1)$. We first claim that $c_{1}=c_{2}=0$ and $c_{3}=\epsilon_{3}= \pm 1$. If $c_{i} \neq 0$, for $i=1$ or 2 , then $2 a_{i}=c_{i}$ by (4.4), $2 b_{i}=c_{i}$ by (4.5) and $2 a_{i} b_{i}=\alpha_{2} c_{i}^{2}$ by (4.3). These equations imply that

$$
4 a_{i} b_{i}=c_{i}^{2}=2 \alpha_{2} c_{i}^{2} .
$$

Because $c_{i} \neq 0$, we have that $1=2 \alpha_{2}$. This gives a contradiction. Therefore, we have

$$
c_{1}=c_{2}=0 .
$$

This together with $\operatorname{det} A_{f}= \pm 1$ imply that

$$
c_{3}=\epsilon_{3}= \pm 1 \text {. }
$$

Because $Z^{2}=-s_{1} X Z-r_{1} Y Z-\alpha_{1} X Y$ in $H^{*}\left(P\left(\eta_{\left(s_{1}, r_{1}, \alpha_{1}\right)}\right)\right)$, the ring isomorphism $f$ induces the following equations

$$
\begin{aligned}
& 2 a_{3} b_{3}-\alpha_{2} \epsilon_{3}^{2}=-s_{1}\left(a_{1} b_{3}+a_{3} b_{1}\right)-r_{1}\left(a_{2} b_{3}+a_{3} b_{2}\right)-\alpha_{1}\left(a_{1} b_{2}+a_{2} b_{1}\right), \\
& \left(2 a_{3}-\epsilon_{3}\right) \epsilon_{3}=\left(-s_{1} a_{1}-r_{1} a_{2}\right) \epsilon_{3}, \\
& \left(2 b_{3}-\epsilon_{3}\right) \epsilon_{3}=\left(-s_{1} b_{1}-r_{1} b_{2}\right) \epsilon_{3} .
\end{aligned}
$$

Using (4.3) and $c_{1}=c_{2}=0$, we have $a_{i} b_{i}=0$ for $i=1,2$. Moreover, from $\operatorname{det} A_{f}= \pm 1$, there are two possibilities, i.e., either $\left(a_{1}, b_{2}\right)=(0,0)$ and $\left(a_{2}, b_{1}\right)=\left(\epsilon_{1}, \epsilon_{2}\right)$, or $\left(a_{1}, b_{2}\right)=\left(\epsilon_{1}, \epsilon_{2}\right)$ and $\left(a_{2}, b_{1}\right)=(0,0)$ where $\epsilon_{i}= \pm 1$ for $i=1,2$.

If $\left(a_{1}, b_{2}\right)=(0,0)$ and $\left(a_{2}, b_{1}\right)=\left(\epsilon_{1}, \epsilon_{2}\right)$, then it follows from (4.7) and (4.8) that

$$
\begin{aligned}
& 2 a_{3}=\epsilon_{3}-r_{1} \epsilon_{1} ; \\
& 2 b_{3}=\epsilon_{3}-s_{1} \epsilon_{2} .
\end{aligned}
$$

It is easy to check that if $s_{1}=0$ or $r_{1}=0$ then we have a contradiction to one of the equations above. Therefore, $\left(s_{1}, r_{1}\right)=\left(s_{2}, r_{2}\right)=(1,1)$. We also have that if $\epsilon_{3}=\epsilon_{1}\left(\right.$ resp. $\left.\epsilon_{3}=\epsilon_{2}\right)$ then $a_{3}=0$ (resp. $b_{3}=0$ ) and if $\epsilon_{3} \neq \epsilon_{1}$ (resp. $\left.\epsilon_{3} \neq \epsilon_{2}\right)$ then $a_{3}=\epsilon_{3}$ (resp. $b_{3}=\epsilon_{3}$ ). Thus, by the equation (4.6), we have that $\alpha_{2}=\alpha_{1}$ or $\alpha_{2}=-\alpha_{1}+1$.

If $\left(a_{1}, b_{2}\right)=\left(\epsilon_{1}, \epsilon_{2}\right)$ and $\left(a_{2}, b_{1}\right)=(0,0)$, then similarly we have that $\left(s_{1}, r_{1}\right)=\left(s_{2}, r_{2}\right)=(1,1)$ and $\alpha_{2}=\alpha_{1}$ or $\alpha_{2}=-\alpha_{1}+1$. This establishes $(3)-(c)$.

Case 2: $\left(s_{2}, r_{2}\right)=(0,0)$. If $\left(s_{1}, r_{1}\right)=(1,1)$ in this case, by the same argument as in Case 1 with $\left(s_{2}, r_{2}\right)$ replaced by $\left(s_{1}, r_{1}\right)$, we can see that $\left(s_{2}, r_{2}\right)=(1,1)$ which contradicts to the hypothesis. Therefore $\left(s_{1}, r_{1}\right)=(0,0)$ or $(1,0)$, and hence, $Z^{2}=-s_{1} X Z-\alpha_{1} X Y$ in $H^{*}\left(P\left(\eta_{\left(s_{1}, r_{1}, \alpha_{1}\right)}\right)\right)$. 
Therefore, the ring isomorphism $f$ implies the following equations:

$$
\begin{aligned}
& 2 a_{3} b_{3}-\alpha_{2} c_{3}^{2}=-s_{1}\left(a_{1} b_{3}+a_{3} b_{1}\right)-\alpha_{1}\left(a_{1} b_{2}+a_{2} b_{1}\right)+s_{1} c_{1} c_{3} \alpha_{2}+\alpha_{1} c_{1} c_{2} \alpha_{2} ; \\
& 2 a_{3} c_{3}=-s_{1}\left(a_{1} c_{3}+a_{3} c_{1}\right)-\alpha_{1}\left(a_{1} c_{2}+a_{2} c_{1}\right) ; \\
& 2 b_{3} c_{3}=-s_{1}\left(b_{1} c_{3}+b_{3} c_{1}\right)-\alpha_{1}\left(b_{1} c_{2}+b_{2} c_{1}\right) .
\end{aligned}
$$

Because of (4.4) and (4.5), we also have that $a_{i} c_{i}=b_{i} c_{i}=0$. Then by (4.3), there are two cases to consider for $i=1,2$ : $(\mathbf{2}-\mathbf{i})$ the case when $c_{i} \neq 0$, and hence, $a_{i}=b_{i}=\alpha_{2}=0$; (2-ii) the case when $c_{i}=0$, and hence $a_{i} b_{i}=0$.

(2-i) If $c_{1} \neq 0$, and hence, $a_{1}=b_{1}=\alpha_{2}=0$, then $c_{1}=\epsilon_{3}= \pm 1$ because $\operatorname{det} A_{f}= \pm 1$. Furthermore, if $c_{2} \neq 0$, then $a_{2}=b_{2}=0$, which gives a contradiction to $\operatorname{det} A_{f}= \pm 1$. Therefore, $c_{2}=0$ and $a_{2} b_{2}=0$. Moreover $a_{3} b_{3}=0$ by (4.9). Since $\operatorname{det} A_{f}= \pm 1$, there are two possibilities for $\left(a_{2}, a_{3}\right)$ and $\left(b_{2}, b_{3}\right)$, i.e., either $\left(a_{2}, a_{3}\right)=\left(0, \epsilon_{1}\right)$ and $\left(b_{2}, b_{3}\right)=\left(\epsilon_{2}, 0\right)$, or $\left(a_{2}, a_{3}\right)=\left(\epsilon_{1}, 0\right)$ and $\left(b_{2}, b_{3}\right)=\left(0, \epsilon_{2}\right)$. If $a_{2}=b_{3}=0$, then, by using (4.10) and (4.11), we have that $2 c_{3}=-s_{1} \epsilon_{3}$ and $\alpha_{1}=\alpha_{2}=0$. Therefore, because $s_{1}=0$ or 1 , we also have $c_{3}=0$ and $s_{1}=s_{2}=0$. If $a_{3}=b_{2}=0$, then we similarly have that $\alpha_{1}=\alpha_{2}=0$ and $s_{1}=s_{2}=0$.

(2-ii) If $c_{1}=0$, then $a_{1} b_{1}=0$. If $c_{2} \neq 0$, then the proof is almost the same with the case when $c_{1} \neq 0$; and we have that $\alpha_{1}=\alpha_{2}=0$ and $s_{1}=s_{2}=0$ as the conclusion. Therefore, we may put $c_{2}=0$ and $a_{2} b_{2}=0$. Because of $\operatorname{det} A_{f}= \pm 1$, we have that $c_{3}=\epsilon_{3}= \pm 1$ and there are the two possibilities, i.e., either $\left(a_{1}, a_{2}\right)=\left(0, \epsilon_{1}\right)$ and $\left(b_{1}, b_{2}\right)=\left(\epsilon_{2}, 0\right)$, or $\left(a_{1}, a_{2}\right)=\left(\epsilon_{1}, 0\right)$ and $\left(b_{1}, b_{2}\right)=\left(0, \epsilon_{2}\right)$. If $a_{1}=b_{2}=0$ (resp. $\left.a_{2}=b_{1}=0\right)$, then it follows from (4.11) (resp. (4.10)) that $2 b_{3}=-s_{1} b_{1}$ (resp. $2 a_{3}=-s_{1} a_{1}$ ). Therefore, $s_{1}=s_{2}=0$ and $b_{3}=0$ (resp. $a_{3}=0$ ). Moreover, by (4.9), we have that $\alpha_{2}=\epsilon_{1} \epsilon_{2} \alpha_{1}$. This establishes $(3)-(a)$.

Case 3: $\left(s_{2}, r_{2}\right)=(1,0)$. In this case, by the same arguments as above, we may assume $\left(s_{1}, r_{1}\right)=(1,0)$, i.e., $Z^{2}=-X Z-\alpha_{1} X Y$ in $H^{*}\left(P\left(\eta_{\left(s_{1}, r_{1}, \alpha_{1}\right)}\right)\right)$. It is sufficient to show that $\alpha_{2}=\alpha_{1}$ or $-\alpha_{1}$. Now, the ring isomorphism $f$ implies the following equations:

$$
\begin{aligned}
& 2 a_{3} b_{3}-\alpha_{2} c_{3}^{2}=-\left(a_{1} b_{3}+a_{3} b_{1}\right)-\alpha_{1}\left(a_{1} b_{2}+a_{2} b_{1}\right)+c_{1} c_{3} \alpha_{2}+\alpha_{1} c_{1} c_{2} \alpha_{2} ; \\
& 2 a_{3} c_{3}-c_{3}^{2}=-\left(a_{1} c_{3}+a_{3} c_{1}\right)-\alpha_{1}\left(a_{1} c_{2}+a_{2} c_{1}\right)+c_{1} c_{3}+c_{1} c_{2} \alpha_{1} ; \\
& 2 b_{3} c_{3}=-\left(b_{1} c_{3}+b_{3} c_{1}\right)-\alpha_{1}\left(b_{1} c_{2}+b_{2} c_{1}\right) .
\end{aligned}
$$

Because of (4.4) and (4.5), we also have $\left(2 a_{i}-c_{i}\right) c_{i}=0$ and $b_{i} c_{i}=0$. By (4.3), if $c_{i} \neq 0$ then $b_{i}=\alpha_{2}=0$ and $c_{i}=2 a_{i}$, and if $c_{i}=0$, then $a_{i} b_{i}=0$.

(3-i) If $c_{1} \neq 0$, then $b_{1}=\alpha_{2}=0, c_{1}=2 a_{1}$. Since $\operatorname{det} A_{f}= \pm 1$, we may put $a_{1}=\epsilon_{1}= \pm 1$. In this case, if $c_{2} \neq 0$ then $b_{2}=0$ and $c_{2}=2 a_{2}$, which contradicts to $\operatorname{det} A_{f}= \pm 1$. Therefore, $c_{2}=0$ and $a_{2} b_{2}=0$. It follows from (4.12) and (4.14) that

$$
2 a_{3} b_{3}=-\epsilon_{1}\left(b_{3}+\alpha_{1} b_{2}\right)=b_{3} c_{3} .
$$

Therefore, there are two cases to consider: the case when $b_{3}=0$, and hence $\alpha_{1} b_{2}=0$; the case when $b_{3} \neq 0$, and hence $c_{3}=2 a_{3}$. If $b_{3} \neq 0$ and $c_{3}=2 a_{3}$, then by $\operatorname{det} A_{f}= \pm 1$ we have $a_{3}=0=c_{3}$ and $b_{3}=\epsilon_{2}= \pm 1$. Then the matrix $A_{f}$ is equal

$$
\left(\begin{array}{ccc}
\epsilon_{1} & 0 & 2 \epsilon_{1} \\
a_{2} & b_{2} & 0 \\
0 & \epsilon_{2} & 0
\end{array}\right) .
$$

This gives a contradiction to $\operatorname{det} A_{f}= \pm 1$. Therefore, $b_{3}=0$, and hence $\alpha_{1} b_{2}=0$. If $b_{2}=0$ then this gives a contradiction to $\operatorname{det} A_{f}= \pm 1$. Hence, we have $b_{2} \neq 0$, and hence $\alpha_{1}=\alpha_{2}=0$.

(3-ii) If $c_{1}=0$ and $c_{2} \neq 0$, then $a_{1} b_{1}=0, c_{2}=2 a_{2}$ and $b_{2}=\alpha_{2}=0$. If $b_{1}=0$, then it is easy to check this gives a contradiction to $\operatorname{det} A_{f}= \pm 1$. Hence, $a_{1}=0$ and $b_{1}= \pm 1$. Because $c_{2}=2 a_{2}$ and $\operatorname{det} A_{f}= \pm 1$, we have $c_{3}-2 a_{3}= \pm 1$. By using (4.13), we also have the equation $c_{3}\left(c_{3}-2 a_{3}\right)=0$. Therefore, $c_{3}=0$, and hence $2 a_{3}= \pm 1$. This gives a contradiction to $a_{3} \in \mathbb{Z}$.

Therefore $c_{1}=c_{2}=0$. Since $\operatorname{det} A_{f}= \pm 1$ and $c_{1}=c_{2}=0$, we can put $c_{3}=\epsilon_{3}= \pm 1$. Then, we can easily see that $a_{1}+2 a_{3}=\epsilon_{3}$ by (4.13) and $b_{1}=-2 b_{3}$ by (4.14). Therefore, by using $a_{1} b_{1}=a_{2} b_{2}=0$ and $\operatorname{det} A_{f}= \pm 1$, we have that $b_{1}=b_{3}=0, b_{2}=\epsilon_{2}= \pm 1$ and $a_{2}=0$, $a_{1}=\epsilon_{1}= \pm 1$. Hence, by using (4.12), we have $\alpha_{2}= \pm \alpha_{1}$. This establishes (3) $-(b)$. Consequently, we have proved the implication $(2) \Rightarrow(3)$. 
Finally, we prove $(3) \Rightarrow(1)$. Consider the diffeomorphism $f=\mathrm{id} \times \operatorname{conj}: \mathbb{C} P^{1} \times \mathbb{C} P^{1} \rightarrow \mathbb{C} P^{1} \times$ $\mathbb{C} P^{1}$ defined by $(p, q) \mapsto(p, \bar{q})$. Because $f$ changes the orientation on $\mathbb{C} P^{1} \times \mathbb{C} P^{1}$, the Euler class $e\left(f^{*} \eta_{(s, r, \alpha)}\right)$ coincides with $-e\left(\eta_{(s, r, \alpha)}\right)$. Because of the definition of Chern class, $e\left(f^{*} \eta_{(s, r, \alpha)}\right)=$ $c_{2}\left(f^{*} \eta_{(s, r, \alpha)}\right)=-c_{2}\left(\eta_{(s, r, \alpha)}\right)=-\alpha$. Because $x$ and $y$ are the first Chern classes of the tautological line bundles of the first and the second factor of $\mathbb{C} P^{1} \times \mathbb{C} P^{1}$, we have $c_{1}\left(f^{*} \eta_{(s, r, \alpha)}\right)=f^{*}(s X+r Y)=$ $s x-r y$. Hence, by Lemmas 2.2 and 4.1 , we have

$$
\begin{aligned}
& f^{*} \eta_{(s, 0, \alpha)} \equiv \eta_{(s, 0,-\alpha)} ; \\
& f^{*} \eta_{(1,1, \alpha)} \otimes \gamma_{2} \equiv \eta_{(1,-1,-\alpha)} \otimes \gamma_{2} \equiv \eta_{(1,1,1-\alpha)},
\end{aligned}
$$

where $\gamma_{2}$ is the pull back of the tautological line bundle over $\mathbb{C} P^{1}$ along the projection $\pi_{2}: \mathbb{C} P^{1} \times$ $\mathbb{C} P^{1} \rightarrow \mathbb{C} P^{1}$ to the second factor. This implies that $P\left(\eta_{(s, r, \alpha)}\right) \cong P\left(\eta_{(s, r,-\alpha)}\right)$ for $(s, r)=(0,0)$ or $(1,0)$ (or $(0,1))$ and $P\left(\eta_{(1,1, \alpha)}\right) \cong P\left(\eta_{(1,1,1-\alpha)}\right)$ for $(s, r)=(1,1)$. This proves the implication $(3) \Rightarrow(1)$.

CASE II: $P\left(\xi_{(s, r, \beta)}\right)$ with the base space $H_{1}$. We prove the cohomological rigidity for $P\left(\xi_{(s, r, \beta)}\right)$ in the following proposition.

Proposition 4.5. The following statements are equivalent.

(1) Two manifolds $P\left(\xi_{\left(s_{1}, r_{1}, \beta_{1}\right)}\right)$ and $P\left(\xi_{\left(s_{2}, r_{2}, \beta_{2}\right)}\right)$ are diffeomorphic.

(2) Two cohomology rings $H^{*}\left(P\left(\xi_{\left(s_{1}, r_{1}, \beta_{1}\right)}\right)\right)$ and $H^{*}\left(P\left(\xi_{\left(s_{2}, r_{2}, \beta_{2}\right)}\right)\right)$ are isomorphic.

(3) Either $\left(s_{1}, r_{1}, \beta_{1}\right)=\left(s_{2}, r_{2}, \beta_{2}\right)$, or one of the following holds:
(a) $\left(s_{1}, r_{1}, \beta_{1}\right)=(0,0, \beta)$ and $\left(s_{2}, r_{2}, \beta_{2}\right)=(0,0,-\beta)(\beta \neq 0)$;
(b) $\left(s_{1}, r_{1}, \beta_{1}\right)=(1,0, \beta)$ and $\left(s_{2}, r_{2}, \beta_{2}\right)=(1,0,-\beta)$;
(c) $\left\{\left(s_{1}, r_{1}, \beta_{1}\right),\left(s_{2}, r_{2}, \beta_{2}\right)\right\}=\{(0,1, \beta),(1,1,-\beta)\}$,

for some $\beta \in \mathbb{Z}$.

By using Proposition 4.4 and 4.5 and Lemma 4.3, we have Theorem 4.2. Let us prove Proposition 4.5 .

Proof. (1) $\Rightarrow(2)$ is trivial. We first prove $(2) \Rightarrow(3)$. By (2.1) we have the isomorphisms

$$
\begin{aligned}
& H^{*}\left(P\left(\xi_{\left(s_{1}, r_{1}, \beta_{1}\right)}\right)\right) \simeq \mathbb{Z}[X, Y, Z] /\left\langle X^{2}, Y^{2}+X Y, Z^{2}+s_{1} Z X+r_{1} Z Y+\beta_{1} X Y\right\rangle, \text { and } \\
& H^{*}\left(P\left(\xi_{\left(s_{2}, r_{2}, \beta_{2}\right)}\right)\right) \simeq \mathbb{Z}[x, y, z] /\left\langle x^{2}, y^{2}+x y, z^{2}+s_{2} z x+r_{2} z y+\beta_{2} x y\right\rangle .
\end{aligned}
$$

Assume there is a ring isomorphism $f: H^{*}\left(P\left(\xi_{\left(s_{1}, r_{1}, \beta_{1}\right)}\right)\right) \simeq H^{*}\left(P\left(\xi_{\left(s_{2}, r_{2}, \beta_{2}\right)}\right)\right)$, and put the matrix representation of $f: H^{2}\left(P\left(\xi_{\left(s_{1}, r_{1}, \beta_{1}\right)}\right)\right) \simeq H^{2}\left(P\left(\xi_{\left(s_{2}, r_{2}, \beta_{2}\right)}\right)\right)$ as

$$
A_{f}=\left(\begin{array}{lll}
a_{1} & b_{1} & c_{1} \\
a_{2} & b_{2} & c_{2} \\
a_{3} & b_{3} & c_{3}
\end{array}\right) .
$$

Note that $\operatorname{det} A_{f}= \pm 1$. Let $\epsilon_{i}= \pm 1(i=1,2,3)$. Because of $X^{2}=0 \in H^{*}\left(P\left(\xi_{\left(s_{1}, r_{1}, \beta_{1}\right)}\right)\right)$, we have

$$
\begin{aligned}
& 2 a_{1} b_{1}-b_{1}^{2}-c_{1}^{2} \beta_{2}=0 \\
& 2 a_{1} c_{1}-c_{1}^{2} s_{2}=0 \\
& 2 b_{1} c_{1}-c_{1}^{2} r_{2}=0 .
\end{aligned}
$$

By using these equations and $\operatorname{det} A_{f}= \pm 1$, it is easy to check that for $\epsilon= \pm 1$

Case 1: if $c_{1} \neq 0$, then there are the following two sub-cases:

- $\left(s_{2}, r_{2}\right)=(0,0)$ with $\left(a_{1}, b_{1}, c_{1}\right)=(0,0, \epsilon)$ and $\beta_{2}=0$;

- $\left(s_{2}, r_{2}\right)=(1,0)$ with $\left(a_{1}, b_{1}, c_{1}\right)=(\epsilon, 0,2 \epsilon)$ and $\beta_{2}=0$,

Case 2: if $c_{1}=0$, then $\left(a_{1}, b_{1}\right)=(\epsilon, 0)$ or $(\epsilon, 2 \epsilon)$.

Because $Y^{2}=-X Y$ in $H^{*}\left(P\left(\xi_{\left(s_{1}, r_{1}, \beta_{1}\right)}\right)\right)$, we also have

$$
\begin{aligned}
& 2 a_{2} b_{2}-b_{2}^{2}-c_{2}^{2} \beta_{2}=-a_{1} b_{2}-b_{1} a_{2}+b_{1} b_{2}+c_{1} c_{2} \beta_{2}, \\
& 2 a_{2} c_{2}-c_{2}^{2} s_{2}=-a_{1} c_{2}-c_{1} a_{2}+c_{1} c_{2} s_{2}, \\
& 2 b_{2} c_{2}-c_{2}^{2} r_{2}=-b_{1} c_{2}-c_{1} b_{2}+c_{1} c_{2} r_{2} .
\end{aligned}
$$


Case 1: $c_{1} \neq 0$. If $\left(s_{2}, r_{2}\right)=(0,0)$, then, by using $(4.16),(4.17)$ and $\left(a_{1}, b_{1}, c_{1}\right)=\left(0,0, \epsilon_{3}\right)$, we can easily show that $a_{2}=b_{2}=0$; however, because $\operatorname{det} A_{f}= \pm 1$, this gives a contradiction. Therefore, $\left(s_{2}, r_{2}, \beta_{2}\right)=(1,0,0)$ and $\left(a_{1}, b_{1}, c_{1}\right)=\left(\epsilon_{1}, 0,2 \epsilon_{1}\right)$. Note that $\operatorname{det} A_{f}\left(a_{2} b_{3}-a_{3} b_{2}\right)$ is the $(1,3)$-entry of the matrix $A_{f}^{-1}$. Therefore, by a similar argument to the above, we can see that if $a_{2} b_{3}-a_{3} b_{2} \neq 0$ then $\left(s_{1}, r_{1}\right)=(1,0)$ and $\beta_{1}=0$. This means that if we get $a_{2} b_{3}-a_{3} b_{2} \neq 0$ then we have $\left(s_{1}, r_{1}, \beta_{1}\right)=\left(s_{2}, r_{2}, \beta_{2}\right)=(1,0,0)$, i.e., the statement of this proposition holds.

By (4.17), we may divide the case when $c_{1} \neq 0$ into two sub-cases: (1-i) $b_{2}=0$ and (1-ii) $b_{2} \neq 0$ and $c_{2}=-\epsilon_{1}$.

(1-i) If $b_{2}=0$, then it easily follows from (4.16) that $c_{2}=2 a_{2}$ or $-\epsilon_{1}$. Moreover, by using $\operatorname{det} A_{f}= \pm 1$ and $\left(a_{1}, b_{1}, c_{1}\right)=\left(\epsilon_{1}, 0,2 \epsilon_{1}\right)$, we have that $\left(a_{2}, b_{2}, c_{2}\right)=\left(0,0,-\epsilon_{1}\right)$ or $\left(-\epsilon_{1}, 0,-\epsilon_{1}\right)$, and $b_{3}=\epsilon_{2}$. If $\left(a_{2}, b_{2}, c_{2}\right)=\left(-\epsilon_{1}, 0,-\epsilon_{1}\right)$, then $a_{2} b_{3}-a_{3} b_{2}=-\epsilon_{1} \epsilon_{2} \neq 0$. Therefore, by the argument explained above, we have $\left(s_{1}, r_{1}, \beta_{1}\right)=\left(s_{2}, r_{2}, \beta_{2}\right)=(1,0,0)$. Hence, this satisfies the statement of this proposition. Suppose $\left(a_{2}, b_{2}, c_{2}\right)=\left(0,0,-\epsilon_{1}\right)$. Since $Z^{2}=-s_{1} X Z-r_{1} Y Z-$ $\beta_{1} X Y$, we have

$$
\begin{aligned}
& \left(2 a_{3} \epsilon_{2}-1\right) x y+2 \epsilon_{2} c_{3} y z+\left(2 a_{3} c_{3}-c_{3}^{2}\right) x z \\
= & -s_{1}\left(\epsilon_{1} x+2 \epsilon_{1} z\right)\left(a_{3} x+\epsilon_{2} y+c_{3} z\right)+r_{1} \epsilon_{1} z\left(a_{3} x+\epsilon_{2} y+c_{3} z\right)+\beta_{1}\left(\epsilon_{1} x+2 \epsilon_{1} z\right) \epsilon_{1} z .
\end{aligned}
$$

So, we have

$$
\begin{aligned}
& 2 a_{3} \epsilon_{2}-1=-s_{1} \epsilon_{1} \epsilon_{2} \\
& 2 a_{3} c_{3}-c_{3}^{2}=-2 s_{1} \epsilon_{1} a_{3}+s_{1} \epsilon_{1} c_{3}+r_{1} \epsilon_{1} a_{3}-r_{1} \epsilon_{1} c_{3}-\beta_{1} \\
& 2 \epsilon_{2} c_{3}=-2 s_{1} \epsilon_{1} \epsilon_{2}+r_{1} \epsilon_{1} \epsilon_{2} .
\end{aligned}
$$

It easily follows from these equations that $\left(s_{1}, r_{1}, \beta_{1}\right)=\left(s_{2}, r_{2}, \beta_{2}\right)=(1,0,0)$.

(1-ii) If $b_{2} \neq 0$ and $c_{2}=-\epsilon_{1}$, then we have that $b_{2}=2 a_{2}+\epsilon_{1}$ by $(4.15)$. Since $\left(a_{1}, b_{1}, c_{1}\right)=$ $\left(\epsilon_{1}, 0,2 \epsilon_{1}\right)$, we have

$$
\operatorname{det} A_{f}=\left(2 \epsilon_{1} a_{2}+1\right)\left(b_{3}+c_{3}-2 a_{3}\right)= \pm 1
$$

Therefore, either (1-ii-a) $\left(a_{2}, b_{2}, c_{2}\right)=\left(0, \epsilon_{1},-\epsilon_{1}\right)$, or $\left(\mathbf{1}\right.$-ii-b) $\left(-\epsilon_{1},-\epsilon_{1},-\epsilon_{1}\right)$ and $b_{3}+c_{3}-2 a_{3}=$ \pm 1 .

(1-ii-a) Suppose $\left(a_{2}, b_{2}, c_{2}\right)=\left(0, \epsilon_{1},-\epsilon_{1}\right)$, then $a_{2} b_{3}-b_{2} a_{3}=-\epsilon_{1} a_{3}$. As before, if $a_{3} \neq 0$ then $\left(s_{1}, r_{1}, \beta_{1}\right)=\left(s_{2}, r_{2}, \beta_{2}\right)=(1,0,0)$. This satisfies the statement of proposition. If $a_{3}=0$, then $b_{3}+c_{3}= \pm 1$ by the equation above. From the relation $Z^{2}=-s_{1} X Z-r_{1} Y Z-\beta_{1} X Y$, we have

$$
\begin{aligned}
& -b_{3}^{2}=-s_{1} \epsilon_{1} b_{3}+r_{1} \epsilon_{1} b_{3}-\beta_{1}, \\
& -c_{3}^{2}=s_{1} \epsilon_{1} c_{3}-r_{1} \epsilon_{1} c_{3}-\beta_{1}, \\
& 2 b_{3} c_{3}=-2 s_{1} \epsilon_{1} b_{3}-r_{1} \epsilon_{1} c_{3}+r_{1} \epsilon_{1} b_{3}-2 \beta_{1} .
\end{aligned}
$$

From these equations, we get

$$
\left(b_{3}+c_{3}\right)^{2}=1=-s_{1} \epsilon_{1}\left(b_{3}+c_{3}\right) .
$$

Hence, $s_{1}=1$ and $b_{3}+c_{3}=-\epsilon_{1}$. By (4.18), we have

$$
-1+2 \epsilon_{1} c_{3}-c_{3}^{2}=-\epsilon_{1}\left(-\epsilon_{1}-c_{3}\right)+r_{1} \epsilon_{1}\left(-\epsilon_{1}-c_{3}\right)-\beta_{1} .
$$

Substituting (4.19) into this equation, we have

$$
-1+2 \epsilon_{1} c_{3}+\epsilon_{1} c_{3}-r_{1} \epsilon_{1} c_{3}-\beta_{1}=-\epsilon_{1}\left(-\epsilon_{1}-c_{3}\right)+r_{1} \epsilon_{1}\left(-\epsilon_{1}-c_{3}\right)-\beta_{1} .
$$

Hence,

$$
2\left(2 \epsilon_{1} c_{3}-1\right)=r_{1}=0 .
$$

But this is impossible. Therefore the case (1-ii-a) can not occur.

(1-ii-b) Suppose $\left(a_{2}, b_{2}, c_{2}\right)=\left(-\epsilon_{1},-\epsilon_{1},-\epsilon_{1}\right)$, then $a_{2} b_{3}-b_{2} a_{3}=-\epsilon_{1}\left(b_{3}-a_{3}\right)$. With the method similar to that demonstrated above, if $a_{3} \neq b_{3}$ then $\left(s_{1}, r_{1}, \beta_{1}\right)=\left(s_{2}, r_{2}, \beta_{2}\right)=(1,0,0)$. 
Hence, we may assume $a_{3}=b_{3}$. Because $\operatorname{det} A_{f}=c_{3}+b_{3}-2 a_{3}= \pm 1$, we also have $c_{3}-b_{3}= \pm 1$. From the relation $Z^{2}=-s_{1} X Z-r_{1} Y Z-\beta_{1} X Y$, we have

$$
\begin{aligned}
& b_{3}^{2}=-s_{1} \epsilon_{1} b_{3}+r_{1} \epsilon_{1} b_{3}+\beta_{1}, \\
& 2 b_{3} c_{3}-c_{3}^{2}=-2 s_{1} \epsilon_{1} b_{3}+s_{1} \epsilon_{1} c_{3}+r_{1} \epsilon_{1} b_{3}+2 \beta_{1}, \\
& 2 b_{3} c_{3}=-2 s_{1} \epsilon_{1} b_{3}+r_{1} \epsilon_{1} c_{3}+r_{1} \epsilon_{1} b_{3}+2 \beta_{1} .
\end{aligned}
$$

By using (4.22) and (4.23), we have

$$
c_{3}\left(r_{1} \epsilon_{1}-c_{3}-s_{1} \epsilon_{1}\right)=0 .
$$

Therefore, we have either $c_{3}=0$, or $c_{3} \neq 0$ and $r_{1} \epsilon_{1}-c_{3}-s_{1} \epsilon_{1}=0$, i.e., $c_{3}=\epsilon_{1}\left(r_{1}-s_{1}\right)$ with $r_{1} \neq s_{1}$.

We claim $c_{3} \neq 0$. If $c_{3}=0$, then by using $\operatorname{det} A_{f}= \pm 1$ and $a_{3}=b_{3}$, we may put $b_{3}=\epsilon_{2}$. By using (4.22) and (4.23) again, we have that

$$
-2 s_{1} \epsilon_{1} \epsilon_{2}+r_{1} \epsilon_{1} \epsilon_{2}+2 \beta_{1}=0 .
$$

Hence, it is easy to check that $\left(s_{1}, r_{1}, \beta_{1}\right)=(0,0,0)$ or $\left(1,0, \epsilon_{1} \epsilon_{2}\right)$. However, using (4.21), both of the cases give contradictions. Consequently, $c_{3} \neq 0$, i.e., $c_{3}=\epsilon_{1}\left(r_{1}-s_{1}\right)$ with $r_{1} \neq s_{1}$.

Because $r_{1} \neq s_{1}$, there are two cases: $\left(s_{1}, r_{1}\right)=(1,0)$ and $(0,1)$. We first assume that $\left(s_{1}, r_{1}\right)=(1,0)$. In this case, $c_{3}=-\epsilon_{1}$. By using (4.22), we have $\beta_{1}=0$. Therefore, this case gives $\left(s_{1}, r_{1}, \beta_{1}\right)=\left(s_{2}, r_{2}, \beta_{2}\right)=(1,0,0)$. We next assume that $\left(s_{1}, r_{1}\right)=(0,1)$. In this case, $c_{3}=\epsilon_{1}$. Similarly, we have that $\epsilon_{1} b_{3}-1=2 \beta_{1}$. This also gives the equation

$$
\epsilon_{1} b_{3}-1=\epsilon_{1}\left(b_{3}-\epsilon_{1}\right)=2 \beta_{1} .
$$

Recall that $b_{3}-c_{3}= \pm 1$ and $c_{3}=\epsilon_{1}$. This gives a contradiction. This finishes Case 1 .

Case 2: $c_{1}=0$. In this case we divided into two sub-cases: (2-i) $\left(a_{1}, b_{1}, c_{1}\right)=\left(\epsilon_{1}, 0,0\right)$, and (2-ii) $\left(a_{1}, b_{1}, c_{1}\right)=\left(\epsilon_{1}, 2 \epsilon_{1}, 0\right)$.

(2-i) Assume $\left(a_{1}, b_{1}, c_{1}\right)=\left(\epsilon_{1}, 0,0\right)$. Then, it follows from (4.15), (4.16) and (4.17) that

$$
\begin{aligned}
& 2 a_{2} b_{2}-b_{2}^{2}-c_{2}^{2} \beta_{2}=-\epsilon_{1} b_{2} ; \\
& 2 a_{2} c_{2}-c_{2}^{2} s_{2}=-\epsilon_{1} c_{2} ; \\
& 2 b_{2} c_{2}-c_{2}^{2} r_{2}=0 .
\end{aligned}
$$

By (4.25) and (4.26), either (2-i-a) $c_{2} \neq 0$ and $2 a_{2}=c_{2} s_{2}-\epsilon_{1}, 2 b_{2}=c_{2} r_{2}$, or (2-i-b) $c_{2}=0$.

(2-i-a) First assume $c_{2} \neq 0$. Then, by $2 a_{2}=c_{2} s_{2}-\epsilon_{1}$, we have $s_{2}=1$ and $c_{2}=2 a_{2}+\epsilon_{1}$. By substituting this equation into (4.26),we have that $r_{2}=0=b_{2}$. Hence, by (4.24), $\beta_{2}=0$, i.e., $\left(s_{2}, r_{2}, \beta_{2}\right)=(1,0,0)$. Because $\operatorname{det} A_{f}= \pm 1$, we may put $b_{3}=\epsilon_{2}$. Moreover, we have $\operatorname{det} A_{f}=$ $-\epsilon_{1} \epsilon_{2}\left(2 a_{2}+\epsilon_{1}\right)= \pm 1$; therefore, $a_{2}=0$ or $-\epsilon_{1}$. If $a_{2}=-\epsilon_{1}$, then $a_{2} b_{3}-a_{3} b_{2}=-\epsilon_{1} \epsilon_{2} \neq 0$. Hence, with the method similar to that demonstrated in Case 1 , we have $\left(s_{1}, r_{1}, \beta_{1}\right)=\left(s_{2}, r_{2}, \beta_{2}\right)=$ $(1,0,0)$. Thus, we may assume $a_{2}=0$, i.e.,

$$
A_{f}=\left(\begin{array}{ccc}
\epsilon_{1} & 0 & 0 \\
0 & 0 & \epsilon_{1} \\
a_{3} & \epsilon_{2} & c_{3}
\end{array}\right) .
$$

By using $Z^{2}=-s_{1} X Z-r_{1} Y Z-\beta_{1} X Y$ and $\left(s_{2}, r_{2}, \beta_{2}\right)=(1,0,0)$, it is easy to get that

$$
\begin{aligned}
& 2 a_{3} \epsilon_{2}-1=-s_{1} \epsilon_{1} \epsilon_{2} ; \\
& 2 \epsilon_{2} c_{3}=-r_{1} \epsilon_{1} \epsilon_{2} ; \\
& \left(2 a_{3}-c_{3}\right) c_{3}=-s_{1} \epsilon_{1} c_{3}-r_{1} \epsilon_{1} a_{3}+r_{1} c_{3} \epsilon_{1}-\beta_{1} .
\end{aligned}
$$

By using the first and second equations, we have $s_{1}=1, r_{1}=0$ and $c_{3}=0$. Therefore, by the third equation, we have that $\left(s_{1}, r_{1}, \beta_{1}\right)=\left(s_{2}, r_{2}, \beta_{2}\right)=(1,0,0)$. Consequently, if $\left(a_{1}, b_{1}, c_{1}\right)=\left(\epsilon_{1}, 0,0\right)$ and $c_{2} \neq 0$, then $\left(s_{1}, r_{1}, \beta_{1}\right)=\left(s_{2}, r_{2}, \beta_{2}\right)=(1,0,0)$. 
(2-i-b) We next assume $c_{2}=0$. Because $\operatorname{det} A_{f}=\epsilon_{1} b_{2} c_{3}= \pm 1$, we may put $b_{2}=\epsilon_{2}$ and $c_{3}=\epsilon_{3}$, i.e.,

$$
A_{f}=\left(\begin{array}{ccc}
\epsilon_{1} & 0 & 0 \\
a_{2} & \epsilon_{2} & 0 \\
a_{3} & b_{3} & \epsilon_{3}
\end{array}\right) .
$$

Then, it follows from (4.24) that $2 a_{2} \epsilon_{2}-1=-\epsilon_{1} \epsilon_{2}$, i.e., $a_{2}=\frac{-\epsilon_{1}+\epsilon_{2}}{2}$. By using $Z^{2}=-s_{1} X Z-$ $r_{1} Y Z-\beta_{1} X Y$, it is easy to get that

$$
\begin{aligned}
& 2 a_{3} b_{3}-b_{3}^{2}-\beta_{2}=-s_{1} \epsilon_{1} b_{3}-r_{1}\left(a_{2} b_{3}+a_{3} \epsilon_{2}-\epsilon_{2} b_{3}\right)-\beta_{1} \epsilon_{1} \epsilon_{2} \\
& 2 b_{3} \epsilon_{3}-r_{2}=-r_{1} \epsilon_{2} \epsilon_{3} \\
& 2 a_{3} \epsilon_{3}-s_{2}=-s_{1} \epsilon_{1} \epsilon_{3}-r_{1} a_{2} \epsilon_{3} .
\end{aligned}
$$

If $\epsilon_{1}=\epsilon_{2}$, then $a_{2}=0$ and

$$
\begin{aligned}
& 2 a_{3} b_{3}-b_{3}^{2}-\beta_{2}=-s_{1} \epsilon_{1} b_{3}-r_{1}\left(a_{3} \epsilon_{1}-\epsilon_{1} b_{3}\right)-\beta_{1} \\
& 2 b_{3} \epsilon_{3}-r_{2}=-r_{1} \epsilon_{1} \epsilon_{3} \\
& 2 a_{3} \epsilon_{3}-s_{2}=-s_{1} \epsilon_{1} \epsilon_{3} .
\end{aligned}
$$

By using the second and third equations, we have that $\left(s_{1}, r_{1}\right)=\left(s_{2}, r_{2}\right)$. Therefore, if $\epsilon_{1}=\epsilon_{3}$, then we also have $b_{3}=a_{3}=0$. Using the first equation, we have $\beta_{1}=\beta_{2}$, i.e., $\left(s_{1}, r_{1}, \beta_{1}\right)=\left(s_{2}, r_{2}, \beta_{2}\right)$. Suppose $\epsilon_{1} \neq \epsilon_{3}$, i.e., $\epsilon_{3}=-\epsilon_{1}$. In this case, if $s_{1}=s_{2}=0$ (resp. $s_{1}=s_{2}=1$ ) then $a_{3}=0$ (resp. $\left.a_{3}=-\epsilon_{1}\right)$ by using the third equation. Similarly by using the second equation, if $r_{1}=r_{2}=0$ (resp. $r_{1}=r_{2}=1$ ) then $b_{3}=0$ (resp. $b_{3}=-\epsilon_{1}$ ). Therefore, by using the first equation, it is easy to check that $\beta_{1}=\beta_{2}$. Consequently, in the case when $\epsilon_{1}=\epsilon_{2}$, hence $\left(a_{2}, b_{2}, c_{2}\right)=\left(0, \epsilon_{1}, 0\right)$, we have $\left(s_{1}, r_{1}, \beta_{1}\right)=\left(s_{2}, r_{2}, \beta_{2}\right)$, i.e., this case satisfies the statement of proposition.

If $-\epsilon_{1}=\epsilon_{2}$, then $a_{2}=-\epsilon_{1}$ and

$$
\begin{aligned}
& 2 a_{3} b_{3}-b_{3}^{2}-\beta_{2}=-s_{1} \epsilon_{1} b_{3}+r_{1} a_{3} \epsilon_{1}+\beta_{1} ; \\
& 2 b_{3} \epsilon_{3}-r_{2}=r_{1} \epsilon_{1} \epsilon_{3} ; \\
& 2 a_{3} \epsilon_{3}-s_{2}=-s_{1} \epsilon_{1} \epsilon_{3}+r_{1} \epsilon_{1} \epsilon_{3} .
\end{aligned}
$$

By using the second equation, we have that $r_{1}=r_{2}$. If $r_{1}=r_{2}=0$, then $b_{3}=0$ by the second equation and $s_{1}=s_{2}$ by the third equation. Moreover, by using the first equation, we have $\left(s_{1}, 0, \beta_{1}\right)=\left(s_{2}, 0,-\beta_{2}\right)$. This implies that $(3)-(a)$ and $(3)-(b)$ in the statement of the proposition. If $r_{1}=r_{2}=1$, then $b_{3}=\frac{\epsilon_{1}+\epsilon_{3}}{2}$ by the second equation and $s_{1} \neq s_{2}$ by the third equation. We first assume $\left(s_{1}, s_{2}\right)=(1,0)$. Then, by the third equation, we have that $a_{3}=0$. Therefore, the first equation gives

$$
-\frac{1+\epsilon_{1} \epsilon_{3}}{2}-\beta_{2}=-\frac{1+\epsilon_{1} \epsilon_{3}}{2}+\beta_{1} .
$$

Therefore, $\beta_{1}=-\beta_{2}$, i.e., $\left(s_{1}, r_{1}, \beta_{1}\right)$ and $\left(s_{2}, r_{2}, \beta_{2}\right)$ are the pair $(1,1, r)$ and $(0,1,-r)$. This implies that $(3)-(c)$ in the statement of the proposition. We next assume $\left(s_{1}, s_{2}\right)=(0,1)$. Then, by the second and third equations, we have that $a_{3}=b_{3}$. Therefore, the first equation gives

$$
\frac{1+\epsilon_{1} \epsilon_{3}}{2}-\beta_{2}=\frac{1+\epsilon_{1} \epsilon_{3}}{2}+\beta_{1} .
$$

Therefore, $\beta_{1}=-\beta_{2}$, i.e., $\left(s_{1}, r_{1}, \beta_{1}\right)$ and $\left(s_{2}, r_{2}, \beta_{2}\right)$ are the pair $(0,1, r)$ and $(1,1,-r)$. This implies that $(3)-(c)$ in the statement of the proposition. Consequently, if $\left(a_{1}, b_{1}, c_{1}\right)=\left(\epsilon_{1}, 0,0\right)$ and $c_{2}=0$, then the statement holds. Therefore the first sub-case (2-i) is done.

(2-ii) Assume $\left(a_{1}, b_{1}, c_{1}\right)=\left(\epsilon_{1}, 2 \epsilon_{1}, 0\right)$. Then, it follows from (4.15), (4.16) and (4.17) that

$$
\begin{aligned}
& 2 a_{2} b_{2}-b_{2}^{2}-c_{2}^{2} \beta_{2}=\epsilon_{1} b_{2}-2 \epsilon_{1} a_{2} ; \\
& 2 a_{2} c_{2}-c_{2}^{2} s_{2}=-\epsilon_{1} c_{2} ; \\
& 2 b_{2} c_{2}-c_{2}^{2} r_{2}=-2 \epsilon_{1} c_{2} .
\end{aligned}
$$

By (4.28) and (4.29), either (2-ii-a) $c_{2} \neq 0$ and $2 a_{2}=c_{2} s_{2}-\epsilon_{1}, 2 b_{2}=c_{2} r_{2}-2 \epsilon_{1}$, or (2-ii-b) $c_{2}=0$. 
(2-ii-a) We first assume $c_{2} \neq 0$. Then, by $2 a_{2}=c_{2} s_{2}-\epsilon_{1}$, we have $s_{2}=1$ and $c_{2}=2 a_{2}+\epsilon_{1}$. Substituting this equation into $2 b_{2}=c_{2} r_{2}-2 \epsilon_{1}$, we have $r_{2}=0$ and $b_{2}=-\epsilon_{1}$. Therefore, $\beta_{2}=0$ by (4.27). By using $Z^{2}=-s_{1} X Z-r_{1} Y Z-\beta_{1} X Y$ and $\left(s_{2}, r_{2}, \beta_{2}\right)=(1,0,0)$, it is easy to get that

$$
\begin{aligned}
& 2 a_{3} b_{3}-b_{3}^{2}=-s_{1}\left(-\epsilon_{1} b_{3}+2 \epsilon_{1} a_{3}\right)-r_{1}\left(a_{2} b_{3}-\epsilon_{1} a_{3}+\epsilon_{1} b_{3}\right)-\beta_{1}\left(1+2 \epsilon_{1} a_{2}\right) ; \\
& 2 b_{3} c_{3}=-2 s_{1} \epsilon_{1} c_{3}-r_{1}\left(-\epsilon_{1} c_{3}+2 a_{2} b_{3}+\epsilon_{1} b_{3}\right)-\beta_{1}\left(4 a_{2} \epsilon_{1}+2\right) ; \\
& \left(2 a_{3}-c_{3}\right) c_{3}=-s_{1} \epsilon_{1} c_{3}-r_{1}\left(-a_{2} c_{3}+2 a_{2} a_{3}+\epsilon_{1} a_{3}-\epsilon_{1} c_{3}\right)-\beta_{1}\left(2 a_{2} \epsilon_{1}+1\right) .
\end{aligned}
$$

Because $\operatorname{det} A_{f}=\left(2 a_{2} \epsilon_{1}+1\right)\left(2 a_{3}-b_{3}-c_{3}\right)= \pm 1$, either (2-ii-a-I) $a_{2}=0$ or (2-ii-a-II) $a_{2}=-\epsilon_{1}$, and we may put $2 a_{3}-b_{3}-c_{3}=\epsilon_{3}$.

(2-ii-a-I) Assume $a_{2}=0$. With the method similar to that demonstrated in Case 1 , if $a_{2} b_{3}-a_{3} b_{2}=a_{3} \neq 0$ then $\left(s_{1}, r_{1}, \beta_{1}\right)=\left(s_{2}, r_{2}, \beta_{2}\right)=(1,0,0)$. Therefore, we may assume $a_{3}=0$ and $-b_{3}-c_{3}=\epsilon_{3}$. Hence, by the above equations, we have that

$$
\begin{aligned}
& -b_{3}^{2}=s_{1} \epsilon_{1} b_{3}-r_{1} \epsilon_{1} b_{3}-\beta_{1} \\
& 2 b_{3} c_{3}=-2 s_{1} \epsilon_{1} c_{3}-r_{1}\left(-\epsilon_{1} c_{3}+\epsilon_{1} b_{3}\right)-2 \beta_{1} ; \\
& -c_{3}^{2}=-s_{1} \epsilon_{1} c_{3}+r_{1} \epsilon_{1} c_{3}-\beta_{1} .
\end{aligned}
$$

This implies that

$$
-\left(b_{3}+c_{3}\right)^{2}=-1=s_{1} \epsilon_{1}\left(b_{3}+c_{3}\right)=-s_{1} \epsilon_{1} \epsilon_{3} .
$$

Therefore, we have $s_{1}=1=\epsilon_{1} \epsilon_{3}$ and $c_{3}=-b_{3}-\epsilon_{1}$. By substituting these equations into the third equation, we have

$$
-b_{3}^{2}-2 \epsilon_{1} b_{3}-1=\epsilon_{1}\left(b_{3}+\epsilon_{1}\right)-r_{1} \epsilon_{1}\left(b_{3}+\epsilon_{1}\right)-\beta_{1} .
$$

Because of the first equation, we have

$$
2 \epsilon_{1} b_{3}+2=r_{1} .
$$

This implies that $r_{1}=0$ and $b_{3}=-\epsilon_{1}$. Hence $c_{3}=-b_{3}-\epsilon_{1}=0$. Therefore, from (4.34), we have $\beta_{1}=0$. Therefore, $\left(s_{1}, r_{1}, \beta_{1}\right)=\left(s_{2}, r_{2}, \beta_{2}\right)=(1,0,0)$. This satisfies the statement of proposition, and the case (2-ii-a-I) is done.

(2-ii-a-II) Assume $a_{2}=-\epsilon_{1}$ With the method similar to that demonstrated in Case 1, if $a_{3} \neq b_{3}$ then $\left(s_{1}, r_{1}, \beta_{1}\right)=\left(s_{2}, r_{2}, \beta_{2}\right)=(1,0,0)$. Therefore, we may assume $a_{3}=b_{3}$ and $a_{3}-c_{3}=\epsilon_{3}$. By the above equations (4.30), (4.31), and (4.32), we have

$$
\begin{aligned}
& a_{3}^{2}=-s_{1} \epsilon_{1} a_{3}+r_{1} \epsilon_{1} a_{3}+\beta_{1} ; \\
& 2 a_{3} c_{3}=-2 s_{1} \epsilon_{1} c_{3}-r_{1}\left(-\epsilon_{1} c_{3}-\epsilon_{1} a_{3}\right)+2 \beta_{1} ; \\
& \left(2 a_{3}-c_{3}\right) c_{3}=-s_{1} \epsilon_{1} c_{3}+r_{1} \epsilon_{1} a_{3}+\beta_{1} .
\end{aligned}
$$

This implies that

$$
\begin{aligned}
\left(a_{3}+c_{3}\right)\left(-a_{3}+c_{3}\right) & =s_{1} \epsilon_{1} a_{3}-s_{1} \epsilon_{1} c_{3}+r_{1} \epsilon_{1} c_{3}-r_{1} \epsilon_{1} a_{3} \\
& =\epsilon_{1}\left(r_{1}-s_{1}\right)\left(-a_{3}+c_{3}\right) .
\end{aligned}
$$

Because $a_{3}-c_{3}=\epsilon_{3}$, we have that $a_{3}+c_{3}=\epsilon_{1}\left(r_{1}-s_{1}\right)$; therefore, $r_{1} \neq s_{1}$. If $\left(s_{1}, r_{1}\right)=(0,1)$, then $2 a_{3} c_{3}=1+2 \beta_{1}$ by the second equation above. This gives a contradiction. Hence, $\left(s_{1}, r_{1}\right)=(1,0)$. In this case, $a_{3}=\frac{-\epsilon_{1}+\epsilon_{3}}{2}$ and $c_{3}=\frac{-\epsilon_{1}-\epsilon_{3}}{2}$. If $\epsilon_{1}=\epsilon_{3}$, then $a_{3}=0$ and $c_{3}=-\epsilon_{1}$. In this case, by using the first equation, $\beta_{1}=0$. However, by using the second equation, we also have $\beta_{1}=-1$. This gives a contradiction and we have $\epsilon_{1}=-\epsilon_{3}$, i.e., $a_{3}=-\epsilon_{1}$ and $c_{3}=0$. It is easy to check that $\left(s_{1}, r_{1}, \beta_{1}\right)=\left(s_{2}, r_{2}, \beta_{2}\right)=(1,0,0)$. Consequently, if $\left(a_{1}, b_{1}, c_{1}\right)=\left(\epsilon_{1}, 2 \epsilon_{1}, 0\right)$ and $c_{2} \neq 0$, then $\left(s_{1}, r_{1}, \beta_{1}\right)=\left(s_{2}, r_{2}, \beta_{2}\right)=(1,0,0)$. This satisfies the statement of proposition. This finishes the proof for (2-ii-a).

(2-ii-b) We next assume $c_{2}=0$, i.e.,

$$
A_{f}=\left(\begin{array}{ccc}
\epsilon_{1} & 2 \epsilon_{1} & 0 \\
a_{2} & b_{2} & 0 \\
a_{3} & b_{3} & c_{3}
\end{array}\right) .
$$


Since $\operatorname{det} A_{f}= \pm 1$, we have $c_{3}= \pm 1=: \epsilon_{3}$. By $(4.27)$

$$
2 a_{2} b_{2}-b_{2}^{2}=\epsilon_{1} b_{2}-2 \epsilon_{1} a_{2} .
$$

Hence,

$$
\left(2 a_{2}-b_{2}\right)\left(b_{2}+\epsilon_{1}\right)=0 .
$$

Therefore, $b_{2}=2 a_{2}$ or $-\epsilon_{1}$. If $b_{2}=2 a_{2}$, then $\operatorname{det} A_{f}=0$, which is contradiction. Therefore, $b_{2}=-\epsilon_{1}$. Hence, $\operatorname{det} A_{f}=\epsilon_{3}\left(-1-2 \epsilon_{1} a_{2}\right)= \pm 1$; therefore,

$$
\begin{aligned}
& \text { - } a_{2}=0 \text { or } \\
& \text { - } a_{2}=-\epsilon_{1} \text {. }
\end{aligned}
$$

By using $Z^{2}=-s_{1} X Z-r_{1} Y Z-\beta_{1} X Y$, it is easy to get that

$$
\begin{aligned}
& 2 a_{3} b_{3}-b_{3}^{2}-\beta_{2}=-s_{1}\left(-\epsilon_{1} b_{3}+2 \epsilon_{1} a_{3}\right)-r_{1}\left(a_{2} b_{3}-\epsilon_{1} a_{3}+\epsilon_{1} b_{3}\right)-\beta_{1}\left(1+2 \epsilon_{1} a_{2}\right) ; \\
& 2 b_{3} \epsilon_{3}-r_{2}=-2 s_{1} \epsilon_{1} \epsilon_{3}+r_{1} \epsilon_{1} \epsilon_{3} ; \\
& 2 a_{3}-s_{2} \epsilon_{3}=-s_{1} \epsilon_{1}-r_{1} a_{2} .
\end{aligned}
$$

By the second equation, we have that $r_{1}=r_{2}$. If $r_{1}=r_{2}=0$, by the second and third equations, we have that $b_{3}=-s_{1} \epsilon_{1}$ and $s_{1}=s_{2}$, respectively. It follows easily from the first equation that $\beta_{1}=\beta_{2}$ for $a_{2}=0$ and $\beta_{1}=-\beta_{2}$ for $a_{2}=-\epsilon_{1}$. This implies that (3) $-(a)$ and (3) $-(b)$ and (3) with $\left(s_{1}, 0, \beta_{1}\right)=\left(s_{2}, 0, \beta_{2}\right)$ in the statement of the proposition. If $r_{1}=r_{2}=1$, then by the above equations, we have that

$$
\begin{aligned}
& 2 a_{3} b_{3}-b_{3}^{2}-\beta_{2}=-s_{1}\left(-\epsilon_{1} b_{3}+2 \epsilon_{1} a_{3}\right)-a_{2} b_{3}+\epsilon_{1} a_{3}-\epsilon_{1} b_{3}-\beta_{1}\left(1+2 \epsilon_{1} a_{2}\right) ; \\
& 2 b_{3} \epsilon_{3}-1=-2 s_{1} \epsilon_{1} \epsilon_{3}+\epsilon_{1} \epsilon_{3} ; \\
& 2 a_{3}-s_{2} \epsilon_{3}=-s_{1} \epsilon_{1}-a_{2} .
\end{aligned}
$$

When $a_{2}=0$, then by the third equation we have that $s_{1}=s_{2}$. If $s_{1}=s_{2}=0$, then by the third equation we have $a_{3}=0$; therefore by the first and second equations we have

$$
-\frac{1+\epsilon_{1} \epsilon_{3}}{2}-\beta_{2}=-\frac{1+\epsilon_{1} \epsilon_{3}}{2}-\beta_{1}
$$

Hence, $\beta_{1}=\beta_{2}$. This implies that (3) with $\left(0,1, \beta_{1}\right)=\left(0,1, \beta_{2}\right)$ in the statement of the proposition. If $s_{1}=s_{2}=1$, then by the second and third equations, we have that $a_{3}=b_{3}=\frac{-\epsilon_{1}+\epsilon_{3}}{2}$. Using the first equation, we have $\beta_{1}=\beta_{2}$. This implies that (3) with $\left(1,1, \beta_{1}\right)=\left(1,1, \beta_{2}\right)$ in the statement of the proposition.

When $a_{2}=-\epsilon_{1}$, then by the third equation we have that $s_{1} \neq s_{2}$. If $\left(s_{1}, s_{2}\right)=(1,0)$, then it follows from the third equation that $a_{3}=0$; therefore by the first and second equations we have

$$
-\frac{1-\epsilon_{1} \epsilon_{3}}{2}-\beta_{2}=-\frac{1-\epsilon_{1} \epsilon_{3}}{2}+\beta_{1}
$$

Hence, $\beta_{1}=-\beta_{2}$. If $\left(s_{1}, s_{2}\right)=(0,1)$, then by the second and third equations, we have that $a_{3}=b_{3}=\frac{\epsilon_{1}+\epsilon_{3}}{2}$. Using the first equation, we have $\beta_{1}=-\beta_{2}$. This implies that $(3)-(c)$ in the statement of the proposition. Consequently, if $\left(a_{1}, b_{1}, c_{1}\right)=\left(\epsilon_{1}, 2 \epsilon_{1}, 0\right)$ and $c_{2}=0$, then the statement holds. Therefore (2-ii-b) is finished, and this establishes the statement $(2) \Rightarrow(3)$.

Finally, we prove $(3) \Rightarrow(1)$. If $\left(s_{1}, r_{1}, \beta_{1}\right)=\left(s_{2}, r_{2}, \beta_{2}\right)$, then the statement is trivial. Assume $\left(s_{1}, r_{1}, \beta_{1}\right) \neq\left(s_{2}, r_{2}, \beta_{2}\right)$. Recall that $H_{1} \cong S^{3} \times_{T^{1}} P\left(\mathbb{C}_{1} \oplus \mathbb{C}\right)$. Let $f: H_{1} \rightarrow H_{1}$ be the diffeomorphism which is induced from the composition of the diffeomorphisms

$$
S^{3} \times_{T^{1}} P\left(\mathbb{C}_{1} \oplus \mathbb{C}\right) \stackrel{g}{\rightarrow} S^{3} \times_{T^{1}} P\left(\mathbb{C}_{-1} \oplus \mathbb{C}\right) \stackrel{h}{\rightarrow} S^{3} \times_{T^{1}} P\left(\mathbb{C}_{1} \oplus \mathbb{C}\right),
$$

where $g$ is the diffeomorphism induced from the orientation reversing of the fibers and $h$ is the diffeomorphism induced from the tensor product of the tautological line bundle on $\gamma_{-1} \oplus \epsilon$. Then, it is easy to check that the induced homomorphism $f^{*}$ is $f^{*}(X)=x$ and $f^{*}(Y)=-x-y$, where $H^{*}\left(H_{1}\right) \simeq \mathbb{Z}[x, y] /\left\langle x^{2}, y^{2}+x y\right\rangle$. Then, we can easily check the following isomorphisms;

$$
\begin{aligned}
& f^{*} \xi_{(0,0, \beta)} \equiv \xi_{(0,0,-\beta)} ; \\
& f^{*} \xi_{(1,0, \beta)} \equiv \xi_{(1,0,-\beta)} ; \\
& f^{*} \xi_{(0,1, \beta)} \equiv \xi_{(-1,-1,-\beta)} .
\end{aligned}
$$


Because of Lemma 2.2, we have

$$
\gamma_{x+y} \otimes \xi_{(-1,-1,-\beta)} \equiv \xi_{(1,1,-\beta)},
$$

where $\gamma_{x+y}$ is the line bundle over $H_{1}$ induced from $x+y \in H^{2}\left(H_{1}\right)$. This establishes that

$$
\begin{aligned}
& P\left(\xi_{(0,0, \beta)}\right) \cong P\left(\xi_{(0,0,-\beta)}\right) ; \\
& P\left(\xi_{(1,0, \beta)}\right) \cong P\left(\xi_{(1,0,-\beta)}\right) ; \\
& P\left(\xi_{(0,1, \beta)}\right) \cong P\left(\xi_{(1,1,-\beta)}\right) .
\end{aligned}
$$

Consequently, using Theorem 3.1 and 4.2, we have Theorem 1.1.

\section{Acknowledgments}

The authors would like to thank Mikiya Masuda for his helpful comments about torus actions on $\mathbb{C} P$-manifolds. The first author would like to give heartful thanks to Prof. Nigel Ray whose comments and helps to stay in University of Manchester were innumerably valuable. He also would like to thank to Prof. Yael Karshon in University of Toronto for giving him an excellent circumstances to do research.

\section{References}

[AtRe] M.F. Atiyah and E. Rees, Vector bundles on projective 3-space, Invent. Math. 35 (1976), 131-153.

[Bo] A. Borel, Sur la cohomologie des espaces fibres principaux et des espaces homogenes de groupes de Lie compacts, Ann. of Math., 57 (1953) 115-207.

[BoHi] A. Borel and F. Hirzebruch, Characteristic classes and homogeneous spaces. I, Amer. J. Math. 80 (1958) $458-538$.

[BoSa] R. Bott and H. Samelson, Applications of the theory of Morse to symmetric spaces, Amer. J. Math. 80 (1958), 964-1029

[BuPa] V.M. Buchstaber and T.E. Panov, Torus Actions and Their Applications in Topology and Combinatorics, University Lecture, 24, Amer. Math. Soc., Providence, R.I., 2002.

[Ch] S. Choi, Classification of Bott manifolds up to dimension eight, arXiv:1112.2321.

[ChKu] S. Choi and S. Kuroki, Topological classification of torus manifolds which have codimension one extended actions, Alg. Geom. Top., 11, (2011), 2655-2679.

[CMS10] S. Choi, M. Masuda, D.Y. Suh, Topological classification of generalized Bott manifolds, Trans. Amer. Math. Soc. 362 (2) (2010) 1097-1112.

[CMS11] S. Choi, M. Masuda, D.Y. Suh, Rigidity Problems in toric topology, a survey, Proc. of the Steklov Inst. of Math., 275, (2011), 177-190; arXiv:1102.1359.

[CPS] S. Choi, S. Park, D.Y. Suh, Topological classification of quasitoric manifolds with the second Betti number 2, arXiv:1005.5431.

[DaJa] M. Davis, T. Januszkiewicz, Convex polytopes, Coxeter orbifolds and torus action, Duke. Math. J., 62 (1991), no. 2, 417-451.

[GrKa] M. Grossberg, Y. Karshon, Bott towers, complete integrability, and the extended character of representations, Duke Math. J., 76 (1994), no. 1, 23-58.

[HaYo] A. Hattori, T. Yoshida, Lifting compact group actions in fibre bundles Japan J. Math. 2 (1976) 13-25.

[Hi] F. Hirzebruch, Über eine Klasse von einfachzusammenhängenden komplexen Mannigfaltigkeiten, Math. Ann., 124 (1951) 77-86.

[Hs] W.Y. Hsiang, Cohomology Theory of Topological Transformation Groups, Ergeb. Math., 85, Springer-Verlag, Berlin, 1975.

[Hu] D. Husemoller, Fibre bundles. Third edition. GTM 20, Springer-Verlag, New York (1994).

[Ka] T. Kaneyama, Torus-equivariant vector bundles on projective spaces Nagoya Math. J., 111 (1988), 25-40.

[KS12] S. Kuroki and D. Y. Suh, Classification of complex projective towers up to dimension 8 and their cohomological rigidity, arXiv:1203.4403.

[KuSu] S. Kuroki and D. Y. Suh, Cohomological non-rigidity of eight-dimensional complex projective towers, preprint.

[Kl] A. Klyachko, Equivariant bundles on toral varieties, Math. USSR Izvestiya 35 2, 337-375 (1990).

[Ma08] M. Masuda, Equivariant cohomology distinguishes toric manifolds, Adv. Math., 218 (2008), 2005-2012.

[Ma10] M. Masuda, Cohomological non-rigidity of generalized real Bott manifolds of height 2, Tr. Mat. Inst. Steklova 268 (2010), Differentsialnye Uravneniya i Topologiya. I, 252-257.

[MaPa] M. Masuda and T.E. Panov, Semi-free circle actions, Bott towers, and quasitoric manifolds, Mat. Sb. 199 (8) (2008) 95-122. 
[MaSu] M. Masuda, D.Y. Suh, Classification problems of toric manifolds via topology, Proc. of Toric Topology, Contemp. Math., 460 (2008), 273-286.

[MiSt] J.W. Milnor, J.D. Stasheff, Characteristic classes, Princeton Univ. Press, 1974.

[MiTo] M. Mimura, H. Toda, Homotopy groups of $S U(3), S U(4)$ and $S p(2)$, J. Math. Kyoto Univ. 3 (1963/1964), 217-250.

[Sh] R.L.E. Schwarzenberger, Vector bundles on algebraic surfaces, Proc. London Math. Soc. 11, 601-622 (1961).

[Wa] C.T.C. Wall, Surgery on compact manifolds, Academic Press, London, 1970. London Math. Soc. Monographs, No. 1.

Osaka City University Advanced Mathematical institute (OCAMi), Osaka, Japan and Department of Mathematics, University of Toronto, Toronto, Canada

E-mail address: kuroki@scisv.sci.osaka-cu.ac.jp/shintaro.kuroki@utoronto.ca

School of Mathematical Science, Korea Advanced Institute of Science and Technology, Daejeon, KOREA

E-mail address: dysuh@math.kaist.ac.kr 